\title{
Cov-MS: A Community-Based Template Assay for Mass- Spectrometry-Based Protein Detection in SARS-CoV-2 Patients
}

Bart Van Puyvelde, Katleen Van Uytfanghe, Olivier Tytgat, Laurence Van Oudenhove, Ralf Gabriels, Robbin Bouwmeester, Simon Daled, Tim Van Den Bossche, Pathmanaban Ramasamy, Sigrid Verhelst, Laura De Clerck, Laura Corveleyn, Sander Willems, Nathan Debunne, Evelien Wynendaele, Bart De Spiegeleer, Peter Judak, Kris Roels, Laurie De Wilde, Peter Van Eenoo, Tim Reyns, Marc Cherlet, Emmie Dumont, Griet Debyser, Ruben t'Kindt, Koen Sandra, Surya Gupta, Nicolas Drouin, Amy Harms, Thomas Hankemeier, Donald J. L. Jones, Pankaj Gupta, Dan Lane, Catherine S. Lane, Said El Ouadi, Jean-Baptiste Vincendet, Nick Morrice, Stuart Oehrle, Nikunj Tanna, Steve Silvester, Sally Hannam, Florian C. Sigloch, Andrea Bhangu-Uhlmann, Jan Claereboudt, N. Leigh Anderson, Morteza Razavi, Sven Degroeve, Lize Cuypers, Christophe Stove, Katrien Lagrou, Geert A. Martens, Dieter Deforce, Lennart Martens, Johannes P. C. Vissers, and Maarten Dhaenens*

Cite This: JACS Au 2021, 1, 750-765

Read Online

ACCESS | Lلll Metrics \& More | 回 Article Recommendations

SI Supporting Information

ABSTRACT: Rising population density and global mobility are among the reasons why pathogens such as SARS-CoV-2, the virus that causes COVID-19, spread so rapidly across the globe. The policy response to such pandemics will always have to include accurate monitoring of the spread, as this provides one of the few alternatives to total lockdown. However, COVID-19 diagnosis is currently performed almost exclusively by reverse transcription polymerase chain reaction (RT-PCR). Although this is efficient, automatable, and acceptably cheap, reliance on one type of

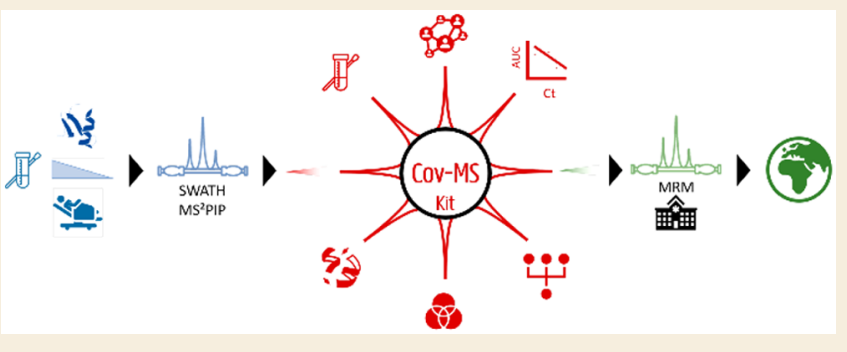
technology comes with serious caveats, as illustrated by recurring reagent and test shortages. We therefore developed an alternative diagnostic test that detects proteolytically digested SARS-CoV-2 proteins using mass spectrometry (MS). We established the Cov-MS consortium, consisting of 15 academic laboratories and several industrial partners to increase applicability, accessibility, sensitivity, and robustness of this kind of SARS-CoV-2 detection. This, in turn, gave rise to the Cov-MS Digital Incubator that allows other laboratories to join the effort, navigate, and share their optimizations and translate the assay into their clinic. As this test relies on viral proteins instead of RNA, it provides an orthogonal and complementary approach to RT-PCR using other reagents that are relatively inexpensive and widely available, as well as orthogonally skilled personnel and different instruments. Data are available via ProteomeXchange with identifier PXD022550.

KEYWORDS: SARS-CoV-2, Mass Spectrometry, Proteomics, Viral Proteins, Diagnostics, MRM

\section{INTRODUCTION}

COVID-19 has disrupted daily life in a substantial part of the world. The virus has forced many countries worldwide to impose lockdowns or take similar wide-ranging measures. To exit such lockdowns, widespread diagnostic capabilities are required to prevent reoccurrence of outbreaks. ${ }^{1}$ Today, millions of reverse transcription polymerase chain reaction (RT-PCR)-based tests are performed every day worldwide. Although these are efficient, relatively simple, and acceptably cheap, reliance on one type of technology comes with notable disadvantages. Not only does this regularly lead to shortages of the necessary reagents and lack of scalable capacity, it also means that these tests are difficult to validate in terms of sensitivity, false positives, and false negative diagnoses. $^{2-4}$ Additionally, nucleic acid alone cannot be used to define viral shedding or infection potential supporting the importance of the detection of other biomolecules. ${ }^{5,6}$ Therefore, numerous publications aim at expanding the viral targets beyond RNA. ${ }^{7-18}$ Like many other viruses, SARS-CoV-2 can produce virus-like particles (VLPs) that contain a high concentration of proteins, but no RNA, making these biomolecules very attractive

Received: February 9, 2021

Published: May 3, 2021 
(a)

(b)

(c)

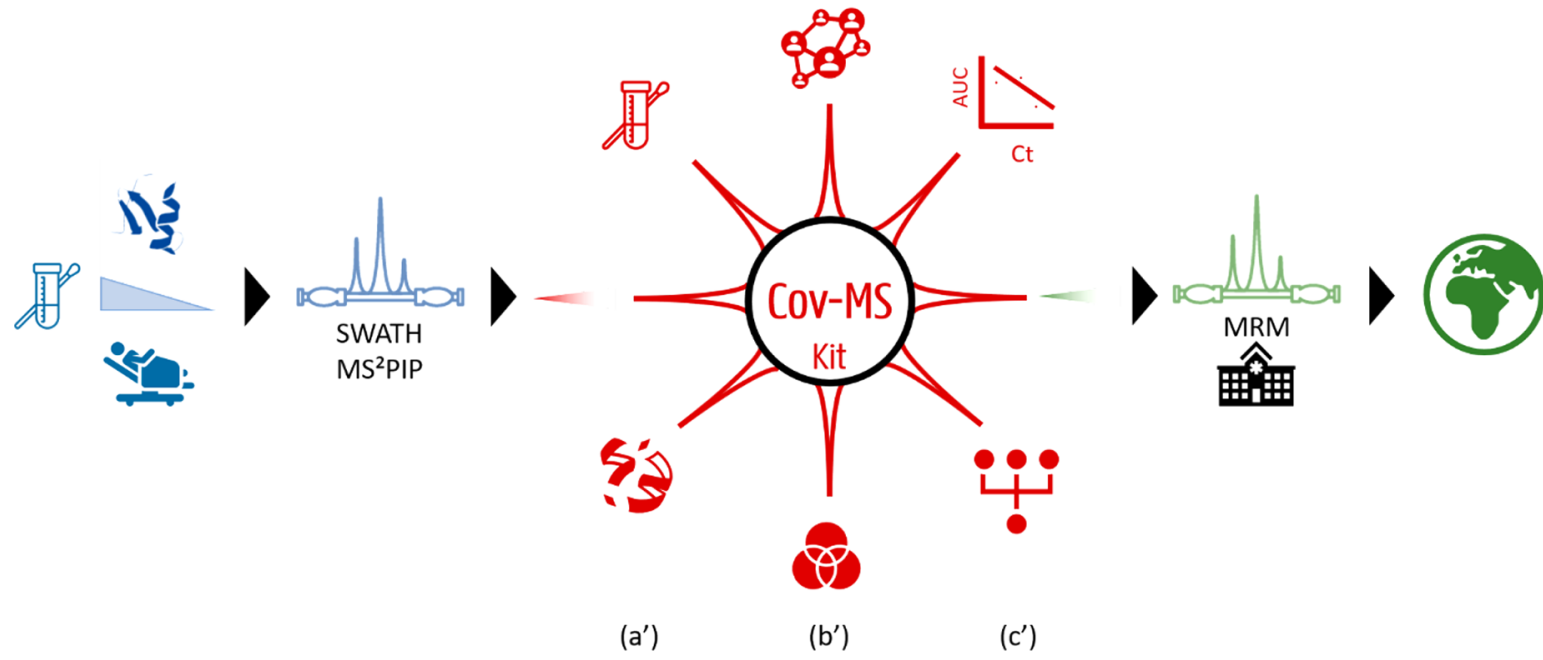

Figure 1. Cov-MS MRM assay development. The SARS-CoV-2 peptide biomarker discovery workflow (blue) was initiated in mid-March 2020. Using the recombinant NCAP and SPIKE protein (Sino Biological, Beijing, China), combined with 20 nasopharyngeal swabs from patients, we applied our recently published data acquisition and data analysis workflow using machine-learning-based spectral predictions. ${ }^{27}$ With the 17 responsive peptides discovered in this way, a preliminary MRM assay was developed that could classify all 20 blinded patient samples correctly. We then assembled the Cov-MS consortium (red) comprising MRM experts from academia and industry, a protein standard company, and a large computational research group. This consortium was provided a Cov-MS kit containing dilution series of recombinant protein digests to optimize instrumental parameters and define their limit of detection (LOD). Optimization of sample preparation assessed alternative transport media and consumables (a) increased digestion efficiency $\left(\mathrm{a}^{\prime}\right)$ and reduced the time investment down to $20 \mathrm{~min}$, and optimization of data acquisition involved 15 different laboratories with different instrumental platforms (b). These were supported by acquisition specialists from three main instrument vendors. By centralizing all data $\left(\mathrm{b}^{\prime}\right)$, instrument-specific peptide targets could be distilled. Optimization of data analysis centered around the correlation between MS signal intensity and clinical RT-PCR assay Ct value (c). Peptides with the highest diagnostic value were filtered out for each medium through machine learning $\left(c^{\prime}\right)$. Additionally, all peptide biomarker candidates were mapped to the 3D structure of the target proteins and investigated for SARS-CoV-2 strain specificity and for known mutations in variants of concern. To enable future clinical roll-out (green), a heavy QConCAT internal standard was synthesized that enables assessing patient sampling quality, sample preparation efficiency, instrumental robustness, and absolute quantification of the viral load. We validated the optimized workflow on 135 patient samples. Finally, we established a Microsoft Teams environment (Cov-MS Digital Incubator) to facilitate global collaboration on the translation of this assay into the clinic. The color-coding in this graphic is used in the rest of the article to indicate in what stage of the development the results should be framed.

targets for liquid chromatography mass spectrometry (LC-MS)based detection and characterization. ${ }^{19-21}$ In fact, MS-based detection of proteins is directly compatible with conventional clinical small molecule detection setups and is adoptable to new mutations in a matter of hours, a very relevant feature in light of the recent D614G variant. ${ }^{22}$ Additionally, the assay is much less prone to contamination than RT-PCR-based assays because of the lack of amplification steps. Thus, in contrast with RT-PCR setups, no pre- and post-PCR lab infrastructure needs to be set up, making a roll-out of an MS-based assay relatively easy in comparison. However, when compared to RT-PCR, MS instrumentation and matrix effects from transport media are more pronounced, and no single working protocol can be expected to be easily implementable across all clinical laboratories.

Therefore, we here describe a community-based effort that has led to a generic and widely applicable MS-based template assay that allows easy adaptation to the broadly variable testing facility landscape (Figure 1). First, we used our high-resolution MS instrument data to create a vendor-independent Skyline document with 17 biomarker peptides from two structural SARS-CoV-2 proteins detected in public data, i.e., nucleocapsid (NCAP_SARS2) and spike (SPIKE_SARS2) protein. ${ }^{17,23} \mathrm{We}$ then established a consortium (MS-Cov), consisting of academic and industrial laboratories, complemented with representation from major MS vendors (Waters Corporation, Sciex, and Thermo Fisher Scientific). All members of this consortium were provided with the Skyline document together with a sample kit containing both pure recombinant NCAP and SPIKE protein as well as a dilution series in a negative patient background. Fifteen laboratories shared data from four different instrument vendors, which was gathered centrally and used in a vendor-independent freeware (Skyline) to assess overall performance and suitability of the candidate signature peptides. $^{24}$ Finally, we have established the Cov-MS Digital Incubator in the form of a Microsoft Teams environment where open communication and data sharing will help translate this assay into different clinical environments around the globe. All supplementary data are available in the Cov-MS Digital Incubator. Access requests can be sent to CovMS@ugent.be.

We here present a very detailed and fully accessible report of the development of a template assay that will enable laboratories worldwide to assess the feasibility of SARS-CoV-2 MS-based detection for their specific platform, navigate and share their optimizations, and quickly adopt the assay in a common effort to significantly increase testing capacity worldwide in the upcoming months. Shortly, we will become part of COVIDMSC. $^{25}$ The LC-MS data generated within the Cov-MS consortium is shared and browsable through Panorama Public (https://panoramaweb.org/CovMS.url); DDA, predicted and chromatogram libraries as well as the narrow window DIA data have been deposited to the ProteomeXchange Consortium via the PRIDE partner repository ${ }^{26}$ with the data set identifier PXD022550 and 10.6019/PXD022550. 

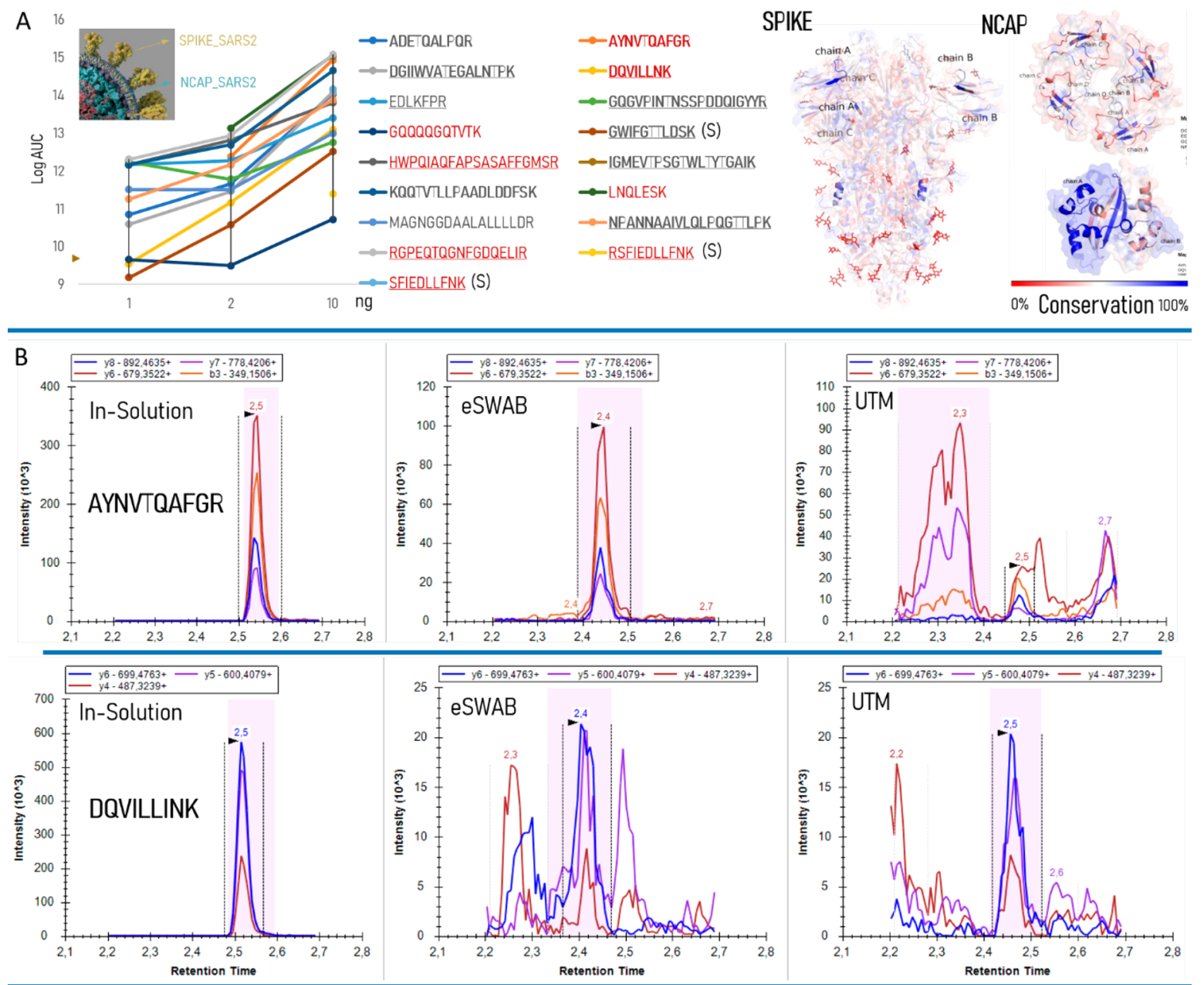

C

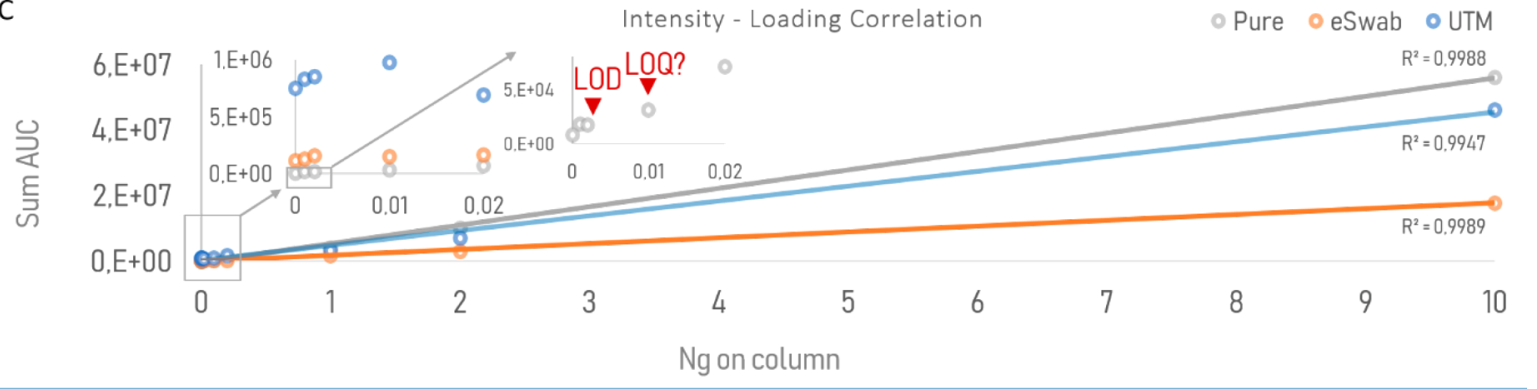

D

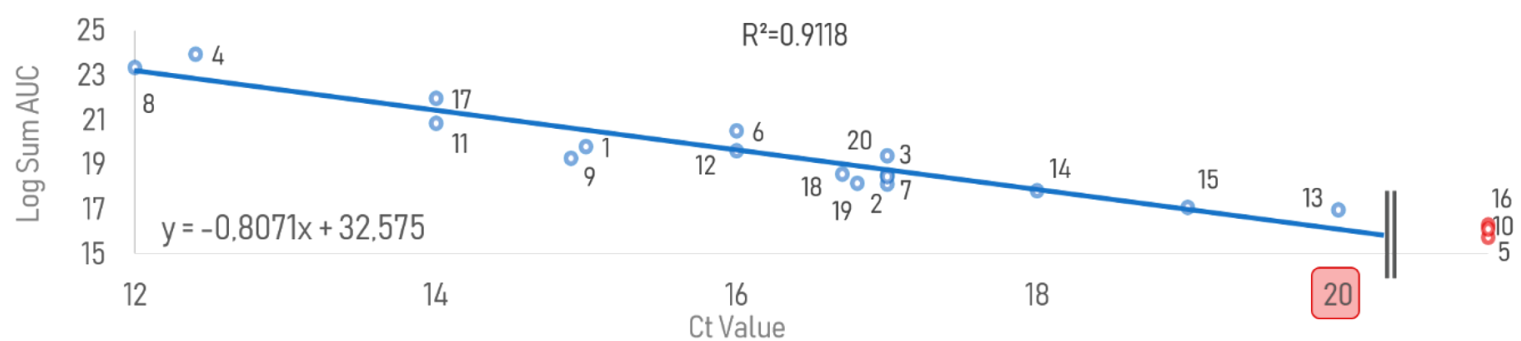

Figure 2. Discovery phase (blue). (A) Seventeen SARS-CoV-2 responsive peptide biomarkers. Based on public data, ${ }^{17,23}$ we selected two target proteins (NCAP_SARS2 and SPIKE_SARS2) and obtained recombinant equivalents (Sino Biological). Using dilution series in a background of 250 $\mu \mathrm{L}$ of UTM from nasopharyngeal swabs of healthy volunteers, we used our recently published workflow combining narrow window DIA with predicted intensities and retention time to detect a total of 17 responsive peptides. ${ }^{27}$ Only intensities of peptidoforms that passed the mProphet threshold of $1 \%$ FDR are depicted. The arrowhead indicates a substituted amino acid in the sequence of the recombinant protein. Bold sequences were also reported in a recent review from Grossegesse et al. ${ }^{18}$ and in ref 21 . Underlined sequences were mapped onto SPIKE_SARS2 (S), NCAP_SARS2 C-terminal dimerization domain and NCAP_SARS2 RNA binding domain (from left to right). Mapped peptides are highlighted in structures, and the 
Figure 2. continued

conservation scores are colored red ( $0 \%$ conserved) to blue (100\% conserved) scale. ${ }^{31}$ Black font indicates sequences unique for the SARS-CoV virus (Detailed Methods section, data analysis). (B) Matrix interference and peptide selection. Irrespective of their evolutionary conservation, all candidate peptides were retained to allow robust assay development compatible with any matrix, experimental condition, or LC-MS instrument used. Depending on the sample conservation buffer, intensities and interferences vary greatly, as illustrated by the transition signals from AYNVTQAFGR and DQVILLINK at $2.5 \mathrm{~min}$ without (in-solution) and with an eSwab and UTM background. Arrowheads indicate correct peak. Signal within dotted lines, i.e., peak boundaries, is summed to calculate the LogSumAUC. (C) Intensity-loading correlation in three different backgrounds. The recombinant proteins were measured in different loading amounts either without matrix (in-solution, $n=1$ ) or in eSwab (saline matrix, $n=1$ ) or UTM (protein matrix, $n=3)(5 \mu \mathrm{L}$ medium equivalent on column). Left inset illustrates the impact of the background on low intensity signal. The right inset highlights the instrumental limit of detection (LOD) and potential limit of quantification (LOQ) of these viral proteins in-solution when all transition intensities are summed. (D) Correlation between MS protein signal and RT-PCR RNA detection. Starting from the Skyline document, an MRM assay was developed for UTM samples on a Xevo TQ-S instrument (Waters Corporation) by MRM transition selection. The final method comprised 10 peptides with a total of 30 transitions. We applied the MRM assay to the 20 patient samples (number coded according to Supplementary Data 3b) in UTM obtained from a University Hospital (Leuven). An equivalent of $5 \mu \mathrm{L}$ (out of $3 \mathrm{~mL}$ ) of medium of each sample was loaded on column. The detection results from the Skyline report were used to logarithmically transform the summed AUC (LogSumAUC) of all the peptides. When plotted against the $\mathrm{Ct}$ value measured by RT-PCR, a strong correlation is found (formula allows conversion of Ct into expected signal), which suggests that this assay can have great potential in the clinic. Negative patients (red) are depicted by their LogSumAUC only beyond the vertical double line on the $X$ axis $(\mathrm{Ct}>40)$.

\section{RESULTS AND DISCUSSION}

Figure 1 depicts a schematic overview of the multiple reaction monitoring (MRM) assay development workflow from discovery to envisioned implementation. Each step is discussed in more detail below in a comprehensive and accessible manner that enables reanalysis of the data online through Panorama Public interface for a broad outreach.

\section{Discovery Phase (Blue): Finding SARS-CoV-2 Peptide Biomarkers}

Sample Preparation. From the start, sample preparation was designed for typical nasopharyngeal swabs, i.e., Copan Universal Transport Medium-RT (UTM-RT). The aim was to minimize sample consumption, time investment, cost, and reagent use and to facilitate parallelization. Therefore, $50 \mu \mathrm{L}$ of UTM was combined with $450 \mu \mathrm{L}$ of ice-cold acetone, resulting in simultaneous protein precipitation and viral-particle neutralization, which allows sample preparation to be performed in a validated biological safety cabinet (BSC) in Biosafety Level L2 (BSL-2) laboratories. ${ }^{28}$ The sample preparation protocol requires only $10 \mathrm{~min}$ of hands-on work and only costs a few euros on reagents. Different grades of trypsin were also compared (Material and Methods and Supporting Information, Detailed Methods, Sample Preparation).

Peptide Biomarker Discovery. The recombinant proteins were analyzed, and a spectral library was created for early assay development based on fractionation of the sample combined with data-dependent acquisition (DDA) and data-independent acquisition (DIA) strategies on high-resolution instruments (Supplementary Data 1). ${ }^{21,29,30}$ A detailed description of this process is presented in Supporting Information (Detailed Methods - Data Analysis - DIA). DIA data are akin to parallel reaction monitoring (PRM) data and in the basic form are identical to what is generated on tandem quadrupole instruments operated in MRM acquisition mode. Therefore, creating a targeted method for any tandem quadrupole instrument becomes trivial, i.e., selecting the transitions that are best detected on a given tandem quadrupole MS instrument in a given lab. We therefore opted to share the assay with the community in this form.

SARS-CoV-2 peptide signals were extracted from the dilution series that were run by a 64 window SWATH on 75 and 20 min LC gradients (Supplementary Data $2 \mathrm{a}$ and $2 \mathrm{~b}$ ) using a highresolution TripleTOF 6600+ Q-TOF instrument (Sciex,
Concord, Canada). We detected 17 responsive peptides that showed an increase in signal with increased loading (Figure 2A and Supplementary Data 2c). At least nine of these have been reported in other studies, confirming that these are indeed interesting targets. ${ }^{18}$ Notably, one peptide (IGMEVTPSGTWLTYTGAIK) from the recombinant protein was not detected due to a single amino acid substitution in the sequence (G16A). Indeed, Grossegesse et al. recently cautioned for the pitfalls of using recombinant proteins for target selection. ${ }^{18}$ Next, data for the 20 blinded patient samples were acquired on a 75 min gradient using the 64 window SWATH mode (Supplementary Data 3a). Through manual inspection of the data, we correctly classified $18 / 20$ samples (Supplementary Data $3 \mathrm{~b})$. The two positive patients that were classified as negative at this point had the lowest viral load according to RTPCR, i.e., with the highest Ct values of 19 and 20. Notably, the exploratory DDA approach had detected SARS-CoV-2 peptides in only two of these 20 patients (Supplementary Data 3c).

Translation into a Targeted MRM Assay with Instant Clinical Applicability. As a template for all downstream optimizations, a Skyline project was created on the DIA data, retaining all 17 peptide biomarkers with 145 transitions (Supplementary Data 4). Notably, two peptides with a missed cleavage were retained because they derive from a $K K, R R, K R$, or RK amino acid sequence motif. ${ }^{32}$ To avoid false positive results, it is important to verify that biomarker peptides are taxonomically uniquely assigned to SARS-CoV-2 (Supplementary Data 5), and to understand the evolutionary conservation of these peptides (Figure 2A). Our taxonomic analysis using Unipept (Supplementary Data 5) illustrates that some of the detected peptides are also expressed in other Coronaviridae or even different organisms. While others have suggested to discard all nonunique sequences during target selection, ${ }^{16,30}$ we maintained these for downstream optimizations. First, a combination of peptides allows higher specificity ${ }^{18}$ and accounts for intrinsic incompleteness of databases. Second, the prevalence of other organisms in nasopharyngeal swabs is not known, and these peptides may therefore never occur apart from SARS$\mathrm{CoV}-2$ infection. Third, variants of concern could easily escape detection if only one or two peptides are being targeted. We therefore mapped amino acid changes of the NCAP protein from www.gisaid.org, which resulted in an exhaustive overview of mutation-prone regions, amino acids, and the continents where they were most prominently found in February 2021 
A

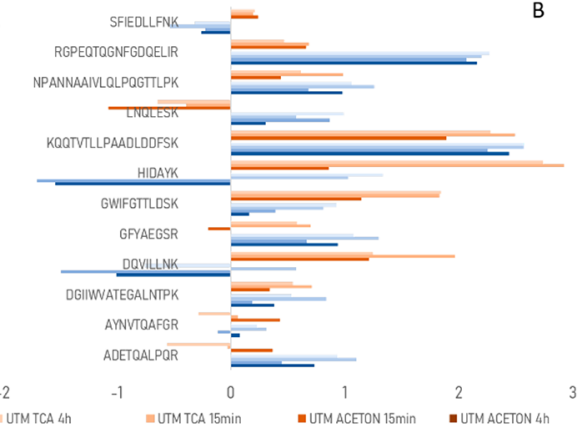

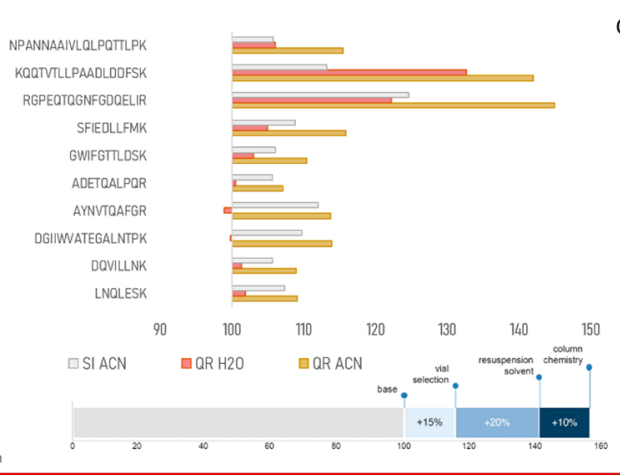

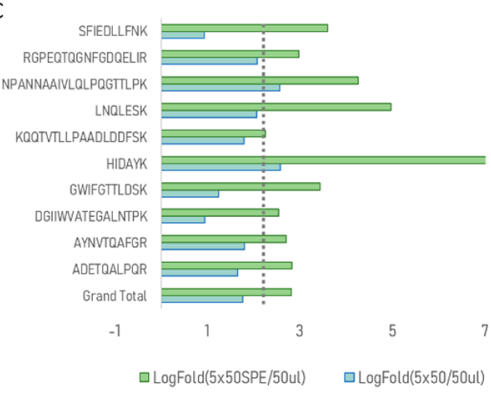

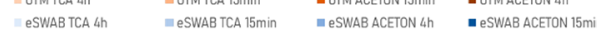

D

$\bigoplus=\frac{\text { AUC }}{\text { Highest } A U C} * 100$

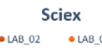

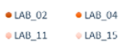

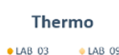

Thermo
- LAB_03 : 4 BB_ 09
Agilent

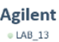

E

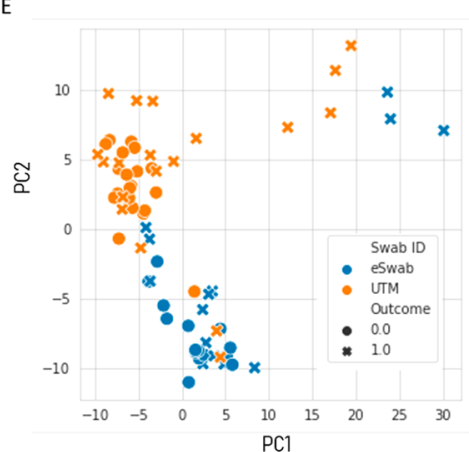

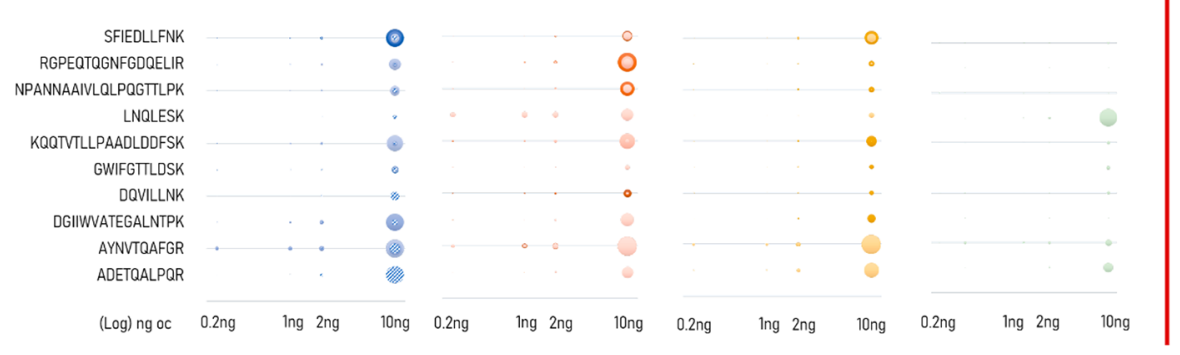

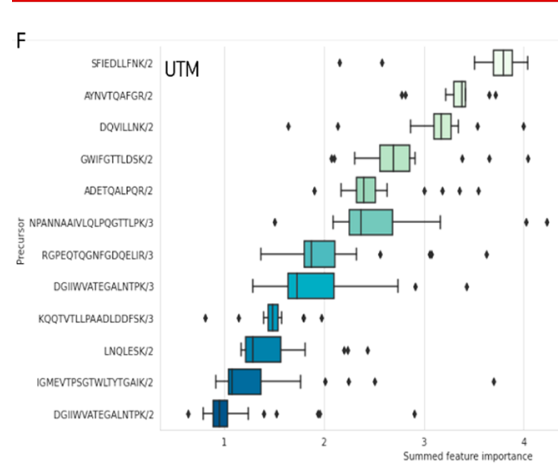

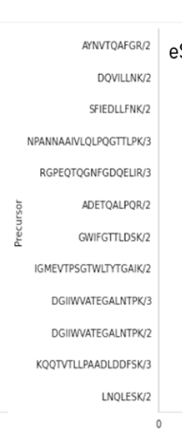

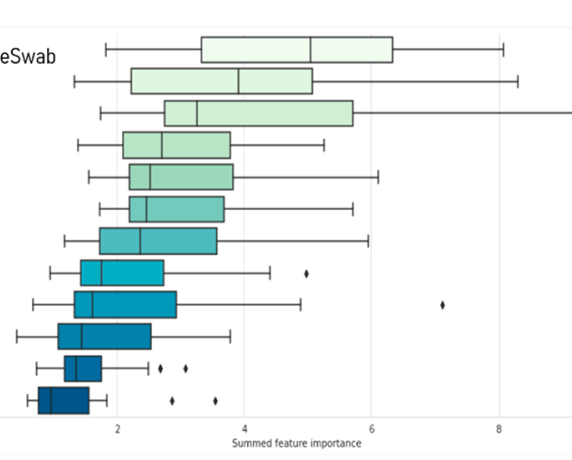

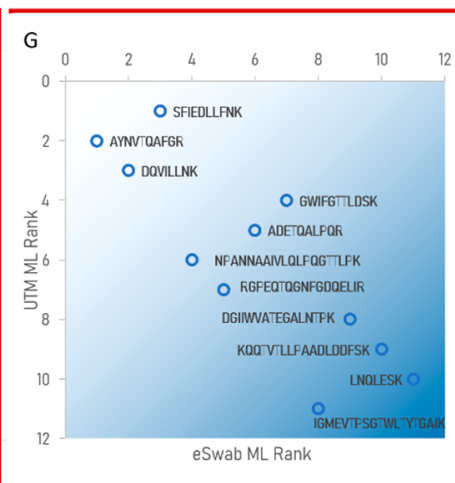

Figure 3. Cov-MS consortium report (red phase). (A) Sample preparation optimization. Different storage media, TCA precipitation, and a 15 min digest $(n=5)$ were compared. The increased LogSumAUC is expressed as log fold changes. (B) Comparing different sample preparation consumables. In the standard workflow, peptides were dissolved in water and transferred to spring insert (SI) sample vials. Using Quan Recovery (QR) vials and adding $5 \%$ acetonitrile (ACN) to the solvent increased the signal for most peptides in solvent but not in UTM (Supplementary Data 10). The bottom bar displays the estimated overall gain attributed to consumables. (C) Increasing the sample load for eSwab samples. Because eSwabs use saline-based preservation solution, increasing the amount of sample on column can be beneficial. Therefore, we assessed the impact of increasing the loading 5-fold, either with or without SPE. An overall average LogSumAUC fold change of 2.3 (dotted line), i.e., 5 times increase in signal, can be attained if the sample is split in five and SPE is applied (LogFold $5 \times 50 \mathrm{SPE}$ ). (D) Data acquisition within the Cov-MS consortium. Fifteen laboratories optimized acquisition on their instrumental platforms using the SOP, the Cov-MS kit, and the Skyline project provided. The bubble plot represents the relative abundance of each peptide in the dilution series compared to the highest abundant peptide for that lab. Strikingly, each lab detected a different (collection of) peptide(s) as the best targets. Several laboratories could detect signal down to $0.2 \mathrm{ng}$ on column in the UTM background. LAB_14 is depicted with shading because this instrument was fitted with a UniSpray source. (E) Optimization of the data analysis. We developed a more datadriven scoring function as an alternative to simple summation of all signal (LogSumAUC). Therefore, a model was trained using all features exported from the Skyline document of 70 patient samples from the AZ Delta sample batch. The principal component analysis (PCA) scoring the distribution of all features illustrates that the conservation medium is one of the most prominent variables in the data set. (F) Peptide feature weighing. The feature weights that are given to each of the MRM transitions by the ML algorithm are representative of their diagnostic value. This in turn can be used to calculate the contribution of each peptide to diagnostic outcome. (G) Final peptide ranking in two different media. From the upper left to the lower right, peptides are depicted by their ML rank in both media. Three peptides could be universally applicable (on a Xevo instrument), irrespective of the medium.

(Supplementary Data 23 and Figure S1a,b). Most importantly, however, compared to RT-PCR, implementation of viral detection MRM assays in the clinic is much more limited by the variability in sampling background (e.g., blood and mucus), conservation medium, and instrument specifications. Therefore, the intensities, retention times $\left(t_{\mathrm{R}}\right)$, and interferences of transitions between different samples can vary greatly when the matrix is not removed. To illustrate this, we depict the signal of AYNVTQAFGR and DQVILLINK, both at a $t_{\mathrm{R}}$ of $2.5 \mathrm{~min}$, as these were measured without matrix (in-solution), in UTM 
(dominant protein background) and in eSwab medium (saline background) with $1 \mathrm{ng}$ on-column (the amount of protein sample analyzed in an LCMS run) (Figure 2B). In conclusion, we recommend targeting all 17 peptides as a starting point in every unique setup where the assay will be implemented. The rollout of an MRM assay is then reduced to omitting those MRM transitions with the poorest performance for that site.

Using this Skyline document, the first MRM assay was optimized on a Xevo TQ-S (Waters Corporation, Milford, MA). It contained 10 peptides, measuring 30 transitions (Detailed Methods section and Supplementary Data 6 and 7). Figure 2C shows that summing the intensities of these 30 transitions (irrespective of individual peak shapes or signal-to-noise) provides a robust metric to quantify dilution series of SARSCoV-2 proteins with and without a matrix. However, the preservation medium has a considerable impact, as shown by background inflation of low signals (in-solution $<\mathrm{eSwab}<$ UTM, Figure 2C left inset) and by matrix suppression of more abundant signals (in-solution > UTM > eSwab). Notably, SPIKE could still be detected down to $70 \mathrm{amol}(10 \mathrm{pg})$ on column and NCAP down to 40 amol (2 pg) on column in solution. This is referred to as the instrumental limit of detection (LOD) (Figure 2C right inset and Supplementary Data 6). In fact, LogSumAUC still allows one to distinguish 0.001 and 0.002 from 0 ng on column, whereas no clear peaks could be annotated in the Skyline project. This implies that summing intensities at specific retention times could be a more robust metric than trying to call single peptide peaks. At $10 \mathrm{pg}$ on column, the summed intensity of all transitions was higher than that of $2 \mathrm{pg}$, making this the potential instrumental limit of quantification (LOQ). Assuming 300 NCAP molecules in a viral particle, as few as 80000 particles can thus theoretically be detected without matrix, in line with previous reports. $9,11,33$

Finally, this optimized MRM assay was applied on the 20 UTM patient samples obtained from University Hospital Leuven, which had been previously diagnosed using RT-PCR. The RT-PCR result is measured by the number of RNA duplication cycles required to detect viral RNA, designated the $\mathrm{Ct}$ value. Therefore, peak boundary corrected transition intensities were summed and Log2-transformed (LogSumAUC) and correlated with the RT-PCR Ct values. From these $5 \mu \mathrm{L}$ UTM on column LC-MS experiments, a strong inverse linear correlation $\left(R^{2}=0.9118\right.$ with the three negative samples excluded) was found between the LogSumAUC from Skyline and the $\mathrm{Ct}$ values measured in the clinic (Figure 2D and Supplementary Data 7). This strong correlation was already suspected $^{18}$ and makes an overwhelmingly strong case for the diagnostic potential of the protein-based MRM assay.

\section{Cov-MS Consortium (Red): Increasing MRM Assay} Sensitivity and Robustness

Based on the observed linear correlation between the measured peptide intensities and the RT-PCR Ct values (Figure 2D), the LOD of the assay, i.e., its sensitivity, was redefined in the context of $\mathrm{Ct}$ values measured in the clinic. This comparison allows improvements to the assay to be expressed in terms of number of $\mathrm{Ct}$ cycles gained, with one $\mathrm{Ct}$ cycle corresponding to a doubling in sensitivity. Indeed, for a larger batch of patient samples $(n=$ 86; $5 \mu \mathrm{L}$ medium equivalent on column) from another hospital (AZ Delta) and that included higher Ct values and different media (Supplementary Data 8), the decrease in LogSumAUC stalled around $\mathrm{Ct} 20$, and there was no difference in measured signal-to-noise $(\mathrm{S} / \mathrm{N})$ at higher $\mathrm{Ct}$ values. Therefore, the next challenge was redefined as increasing the $\mathrm{S} / \mathrm{N}$ in order to gain as many $\mathrm{Ct}$ values as possible, i.e., to gain detection doublings. In essence, this implied straightening of the curve (Figure 3 ).

On April 21, 2020, we presented our discovery phase and preliminary results online to over 80 interested parties to establish a consortium (Cov-MS) to broaden the scope of the work, transfer the methods to other (vendor) instruments, and bring in the expertise of other functions and scientists. ${ }^{34}$ This allowed parallelization of the sample preparation, data acquisition, and data analysis optimization (Figure 1).

Sample Preparation Optimizations: 2-3 Ct Values. Efforts focused around three optimizations: applying a different precipitation strategy with acid, i.e., trichloroacetic acid (TCA) and hydrochloric acid $(\mathrm{HCl})$, reducing digestion time to $15 \mathrm{~min}$, and using different conservation media. While many of these changes increased the overall signal (Figure 3A and Supplementary Data 9), the best protocol is typically dependent on the MRM assay under development as many changes are peptidespecific and might be attenuated by increased ion suppression or noise depending on the medium, sample-related specifics, and experimental conditions. Nevertheless, the reduction of the digestion protocol to only $15 \mathrm{~min}$ (with Promega Trypsin-Lys-C Mix, mass spec grade) now enables a complete sample preparation workflow of less than $30 \mathrm{~min}$.

In parallel, the use of different LC columns, sample vials, and resuspension protocols were also investigated, alongside peptide stability (Figure 3B and Supplementary Data 10). Beneficial effects were incremental, on the order of $10-20 \%$ gains in LogSumAUC for (i) using 5\% acetonitrile for redissolving the peptides, (ii) using dedicated sample containers (QuanRecovery Vials, Waters Corporation, Milford, MA), and (iii) reversedphase separation columns to minimize nonspecific binding (ACQUITY PREMIER Peptide BEH C18 Column, $300 \AA$, 1.7 $\mu \mathrm{m}, 2.1 \times 50 \mathrm{~mm}$, Waters Corporation, Milford, MA). Notably, the gain is lower in UTM background, most probably due to carrier protein effects in this medium.

Crucially, the biggest limitation in terms of sensitivity is the amount of sample that can be loaded on column. More specifically, the results from the discovery phase (blue) were all attained on $5 \mu \mathrm{L}$ medium equivalent on column. Unfortunately, increasing sample load using solid-phase extraction is not straightforward for MRM assays. A detailed description on the main concerns can be found in the Detailed Methods section. Still, the fold changes of each peptide are shown in the anticipated 5-fold increase in signal (dotted line); that is, a minimum of two $\mathrm{Ct}$ values can be attained if five times $50 \mu \mathrm{L}$ digest solution is concentrated onto a single SPE column (Figure 3C and Supplementary Data 11). Automation will allow this finding to be consolidated and extended, yet we caution here for SPE overloading. Given the lack of in-house automation, we continued with the original protocol on a $50 \mu \mathrm{L}$ sample and 10 $\mu \mathrm{L}$ injection on column without applying SPE.

In conclusion, all validated sample preparation strategies have a peptide-specific effect. Therefore, it is worth re-evaluating these steps once the final peptide selection for an assay has been made.

Data Acquisition Optimization: 1-2 Ct Values. After the online presentation of the assay on April 21st, 2020, many of the attendees joined the Microsoft Teams Cov-MS team then containing three channels, one per participating vendor (Waters Corporation, Sciex, and Thermo Fisher Scientific). At the time of submission, the group counted over 100 participants. The Cov-MS laboratories were encouraged to discuss method 


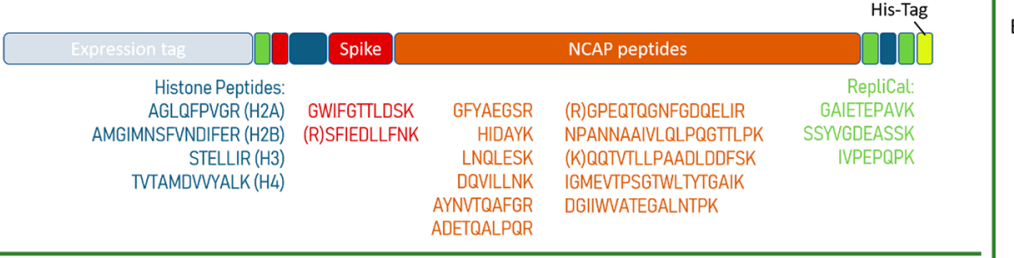

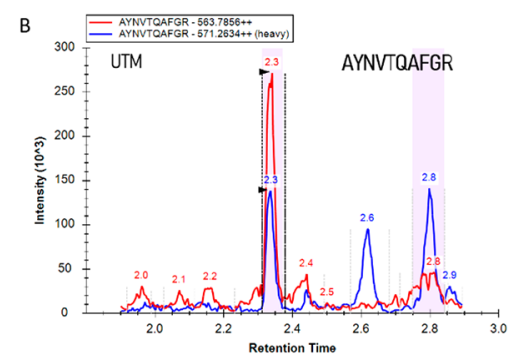
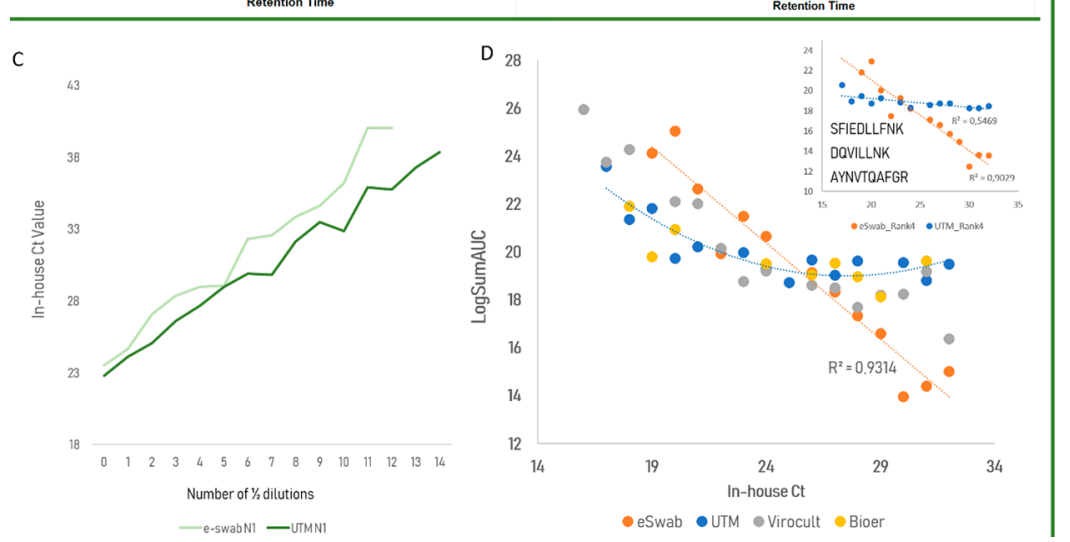

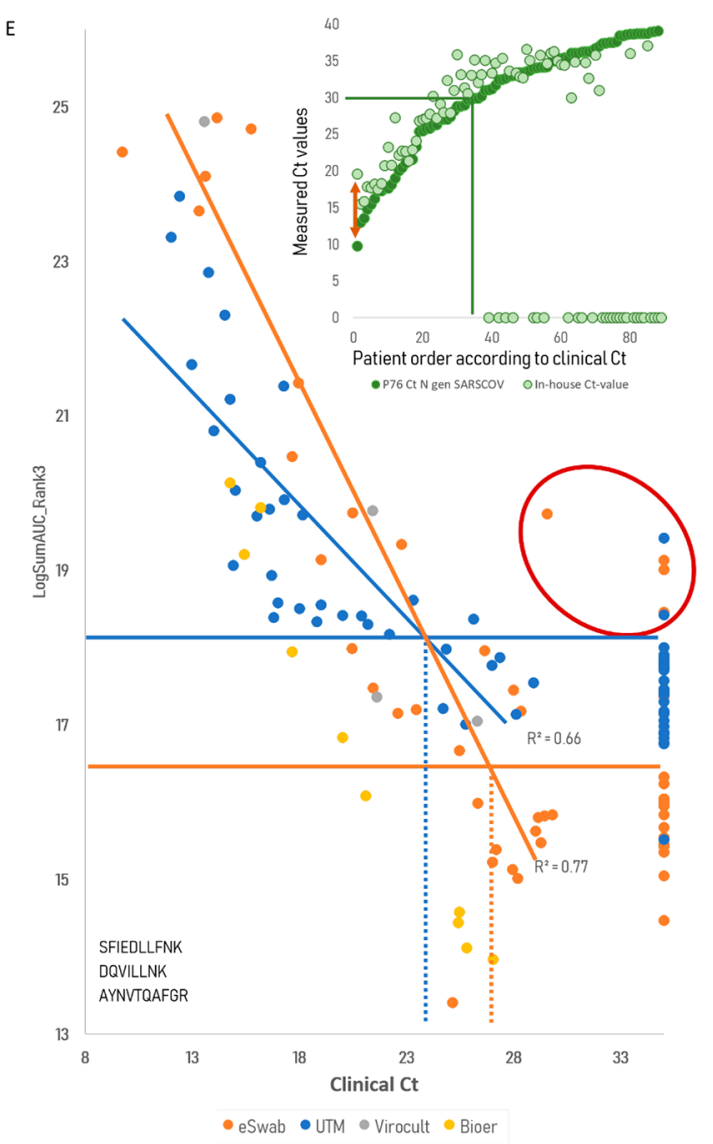

Figure 4. Toward translating the Cov-MS assay into the clinic (green phase). (A) Cov-MS QConCAT internal standard. An isotope labeled ("heavy") construct was expressed in E. coli to troubleshoot and standardize assay development. It contains the 17 stable-isotope-labeled (SIL) SARS-CoV-2 peptides for absolute quantification (red and orange), three RePLiCal peptides to assess LC system stability (green), and four host peptides derived from histones to assess the efficiency of the swab sampling procedure (dark blue). (B) Facilitating peak detection using the Cov-MS internal standard. The two peptides from Figure 2B are depicted in red in their most interfering matrix. The blue trace depicts the heavy signal from the Cov-MS SIL (2 ng and $2.5 \mathrm{ng}$ on column), showing how peak detection now becomes automatable. (C) In-house Ct values for a dilution series of viral particles from the National Reference Center in Belgium. We generated a dilution series in two different backgrounds and determined the Ct values using our inhouse RT-PCR assay. eSwab consistently gave higher Ct values compared to Copan UTM-RT in our hands, but the detection limits attained would allow for accreditation of our RT-PCR workflow. (D) Patient dilution series as an alternative assessment procedure. The viral particle used in the dilution series presented in (C) did not yield any reliable signal in the MRM assay. Therefore, four patients with comparable in-house Ct values in four different backgrounds were selected and diluted in their respective background of negative patients. The $X$-axis depicts the theoretical $\mathrm{Ct}$ values in such dilution series according to (C) and the $y$-axis represents the LogSumAUC of the 13 peptides retained in this MRM assay (Supplementary Data 19). The Cov-MS QConCAT was not yet included in this experiment. Most notably, eSwab retains a linear correlation with intensity below a LogSumAUC of 18, which is not noticeable in the other media. The inset shows the results when only the best three peptides from the ML (Figure 3G) are used for eSwab and UTM. (E) Large patient cohort. Inset: 89 clinically positive patients (dark green) were first selected for in-house RT-PCR (light green), and both $\mathrm{Ct}$ values were compared. Negative in-house results are depicted on the $X$-axis. The arrow (inset) highlights the discrepancy between the eSwab clinical and in-house $\mathrm{Ct}$ values for the patient used for the dilution series in (D). All patients with a clinical Ct below 30 were positive in both assays and were retained for MRM assay validation $(n=82)$. Using the best three peptides from the ML (Figure 3G), the LogSumAUC was plotted against the clinical Ct value. Negative patients are depicted to the right to sample the noise. The outliers are circled in red. The horizontal line shows the LogSumAUC_Rank3 below which signal cannot be confidently distinguished from noise in UTM (blue) and eSwab (orange). For both media, the cross section with the linear regression line is projected downward to estimate the theoretical sensitivity in terms of Ct value.

optimization with the vendors' international expert support teams.

To streamline the integration of all these parallel optimizations, consortium members were provided a detailed standard operating procedure (SOP) for method optimization, the Skyline template containing the 17 peptides (145 transitions), and a Cov-MS kit (Supplementary Data 12). These kits contained digests of pure recombinant NCAP and SPIKE protein as well as a dilution series in a UTM background from healthy donors, in triplicate. The kits allow members to standardize the optimization protocol, to assess performance under different circumstances, and to centralize all data to distill best practices per vendor. Figure $3 \mathrm{D}$ summarizes overall peptide detectability for 15 participating laboratories. Individual instrumental platform data are provided in Supplementary Data 13 and 14 in an easily handled pivot table format. Data can be browsed directly (https://panoramaweb.org/CovMS.url) in Panorama Public. Several partners compiled detailed reports, and Sciex and Waters Corporation published several application notes based on their data ${ }^{35-38}$ (Supplementary Data 15).

Importantly, only the latest generation tandem quadrupole instruments can reach adequate sensitivity. Additionally, the compiled results confirm the initial hypothesis that peptide selection is lab-specific. This was recently also described in a 
review on currently available MS-based detection assays. ${ }^{18}$ However, because the data in this study were acquired on the same samples and analyzed using the same software, it is possible to draw direct conclusions on the acquisition itself. These preliminary results imply that different vendors could benefit from different sets of target peptides, even if the same transitions were targeted (Figure 3D). One striking example is the RGEPQTQGNFGDQELIR peptide, which is especially prominent on Sciex instruments, possibly because the corresponding ion sources are better suited to higher charged peptides. The impact of the source is best illustrated by LAB_14 that used a UniSpray source (Waters Corporation) instead of an ESI source, which boosts the ionization of the ADETQALPQR peptide compared to all other laboratories. ${ }^{37}$ The latter is especially interesting in light of a previous report that argues for this target peptide. ${ }^{18,39}$ While this remains to be fully confirmed by the growing Cov-MS consortium and beyond, including ionization efficiencies in MALDI sources, the drafting of vendor-specific best practices will likely be important for streamlined lab-specific assay rollout. As a starting point, four different technical notes from two vendors are already published. ${ }^{35-38}$

Data Analysis Optimization: 1-2 Ct Values. Figure 2D depicts the quantitative MRM results as the LogSumAUC of all ions within the peak boundaries of the transitions in that particular assay. The initial selection of these transitions was based on an expert's choice from the Copan UTM-RT dilution series in the Cov-MS kit. However, not all transitions contribute to diagnosis equally, and excluding those transitions that are subject to interference in a specific matrix, have low signal, or are simply redundant allows retention of the best transitions in the final LogSumAUC and increased instrumental dwell times to boost sensitivity. However, because of the high variability, this can only be attained by a data-driven approach.

To construct such a data-driven scoring function to distinguish between positive from negative COVID-19 patients from the MRM data, a machine learning (ML) model was trained and evaluated (Supporting Information, Detailed Methods). The training and evaluation data consisted of a Skyline document export of 70 LC-MS experiments on a Xevo TQ-S (Waters Corporation) accompanied by relevant metadata: healthy or diseased, medium used, and $\mathrm{Ct}$ as determined by RT-PCR at the time of initial diagnosis (Supplementary Data 8). The full script and the weights of the individual transitions is available in Supplementary Data 16 and on github (https:// github.com/compomics/Cov-MS-scoring).

The principal component analysis of the feature scores exported from Skyline clearly illustrates that the medium is the most prominent contributor to the second principal component and thus to the choice of transitions and the attainable diagnostic value (Figure 3E). Therefore, in a second round, we trained the two patient populations separately (eSwab, $n=$ 29 , and UTM, $n=41$ ). Figure 3 F depicts the diagnostic weight of each peptide in the respective media.

By plotting the order in which the peptides appear in both media a common set of three peptides surfaced that appears to be well suited for both Copan UTM-RT and eSwab transport media. Even more excitingly, these three peptides cover both SPIKE and NCAP structural proteins (Figure 2A), making this a very attractive target selection (Figure $3 \mathrm{G}$ ).

In conclusion, all parts of the workflow impact each peptide differently. While there is great agreement on the topmost applicable targets, the final order in which a lab can detect them still shifts considerably. Therefore, standardization will be essential for any MS-based assay, and each platform will target different sets of peptides, akin to the use of different primers in PCR laboratories.

\section{Toward a Clinical MRM Assay (Green)}

From the start, our joint efforts have focused on fast and efficient clinical applicability. Therefore, we retain the matrix, reduce sample preparation to below $30 \mathrm{~min}$, provide kits, SOPs and software methods, and only use tandem quadrupole instruments in configurations that are readily available in the clinic. Finally, we describe the first steps in standardization and validation of the assay for clinical implementation and present our Cov-MS Digital Incubator to facilitate communication and exchange on further improvements.

Cov-MS Internal Standard Assesses Sampling Efficiency, Sample Preparation, Data Acquisition, and Data Analysis Automation. In clinical MS-based assays, stableisotope-labeled (SIL) internal standards are indispensable to meet measurement accuracy and to satisfy regulatory requirements. For this assay, together with PolyQuant, we designed the Cov-MS QConCAT, i.e., an artificial protein comprising concatenated peptides to allow for absolute quantification ${ }^{40}$ (Figure 4A). It not only allows to report absolute viral load and interlaboratory comparison, it also enables validation of the entire workflow: (i) swab sampling procedure efficiency in the clinic, (ii) sample preparation efficiency, and (iii) instrument stability, all while (iv) greatly enhancing automation potential of the data analysis. Briefly, PolyQuant (Bad Abbach, Germany) joined the Cov-MS consortium and initiated the expression of a QConCAT protein construct inE. coli that contains the 17 peptide biomarkers. These are heavy labeled through the incorporation of ${ }^{15} \mathrm{~N}$ and separated by their native amino acid context to assess the efficiency of the digest. Additionally, three RePLiCal (i.e., a QconCAT protein for retention time standardization in proteomics studies) retention time standard peptides were incorporated to help assess LC stability. ${ }^{41}$ Finally, four peptides, one from each of the human core histone proteins, were added to the labeled construct to serve as host protein markers for swab sampling quality. We reasoned that nuclear proteins will only be present if the swab caused superficial tissue damage to release intracellular viral particles from cells. Therefore, when histones are low or absent, this could point toward poor swab sampling efficiency and thus a potential falsenegative result. Indeed, all 20 patients from Figure $2 \mathrm{D}$ contained at least one of these four histone peptides in the DDA runs (Supplementary Data 3C and 17). Therefore, the MS-Cov QconCAT construct will help in end-to-end assay troubleshooting and enables the direct comparison of results from all over the world. Note that QconCAT is as scalable as SIL peptides, albeit at a higher cost. Also, in order to include variants of concern, a new construct needs to be expressed.

A Skyline document comprising both "heavy" (from the QcontCAT protein) and "light" (from the native viral and human proteins) transitions was compiled, and three on column loadings were compared for both UTM and eSwab (Supplementary Data 17). Figure $4 \mathrm{~B}$ shows the compiled signal of the light and heavy form of the two peptides from Figure $2 \mathrm{~B}$ in their most interfering matrices. It is clear that the heavy signal (detected here by one transition) greatly facilitates correct peak detection. The discussion on peak detection automation in Skyline can be followed in the Skyline support discussion thread. ${ }^{42}$ Notably, $1.25 \mathrm{ng}$ was loaded in the final patient batch to avoid native peptide ion suppression, but this turned out not 
to be enough, especially in Copan UTM-RT background. Additionally, it might prove beneficial in the future to target more transitions of the heavy and light peptides to facilitate peak detection and retain the dotp value as an additional feature in the Skyline report. Finally, we noted that nearly all peptides (from the QConCAT) display a forward retention time shift in Copan UTM-RT background compared to eSwab (Supplementary Data 17).

RT-PCR Accreditation Standard and Ct Values Are Not Easily Transposable to an MRM Assay. For external quality assessment, we requested positive control material from the National Reference Center (NRC) in Belgium. Developed for RT-PCR, this purified heat-inactivated virus is used to create a dilution series that allows laboratories to determine their LOD. These are series of $1 / 2$ dilutions, each thus corresponding to one Ct cycle. The starting solution (dilution 0 ) was said to be the equivalent of a Ct 17 in the hands of the NRC (Supplementary Data 18). By simply applying the formula of Figure 2D, a linear correlation with their patient $\mathrm{Ct}$ values would allow us to easily detect the first few dilutions, i.e., by a predicted LogSumAUC of 19.3. However, because different RT-PCR assays can vary up to orders of magnitude in the number of copies that can be detected, ${ }^{3}$ we first developed an in-house RT-PCR assay using plasmids as targets. Applying the manufacturer's instructions, we were able to detect down to 10 copies of the $\mathrm{N}$-gene plasmids at a Ct of 38, which corresponds to 2.5 copies at $\mathrm{Ct} 40$, the theoretical upper limit for RT-PCR diagnosis (Supplementary Data 18).

We then measured this NRC dilution series in negative patient background with our in-house RT-PCR assay and could detect the $\mathrm{N} 1$ gene after $10(\mathrm{Ct} 36)$ and $14(\mathrm{Ct} 38) 1 / 2$ dilutions in eSwab and UTM, respectively, which would allow for accreditation (Figure 4C and Supplementary Data 18). However, this still was 5-6 Ct values higher than in the hands of the NRC. Additionally, several attempts to detect a peptide signal in these standards by means of LC-MS failed. This is most surprising given the fact that the patients in Figure 2D were analyzed with $5 \mu \mathrm{L}$ medium equivalent on column and that we now loaded up to $100 \mu \mathrm{L}$ medium equivalent for these standards using an optimized acquisition and sample preparation strategy. We attribute this outcome to (a combination of) any of the following reasons: (i) sample degradation (both mRNA and protein), (ii) specific in-house sample preparation steps being incompatible for the specific purpose of detecting proteins in these purified viral particle standards, (iii) the heat inactivation step, or (iv) the potential role played by viral-like particles (VLP), ${ }^{19,20}$ which were not present in these ultracentrifuge purified standards. In other words, in patient samples, a higher signal for proteins could be present than would be theoretically anticipated from the RT-PCR positive control set. This implies that proficiency assessment schemes for this assay will only be possible through an alternative assessment procedure (AAP), as is often the case when different biomolecules are targeted in clinical assays (see section 9.1.1 from CLSI 62-A).

We therefore propose patient dilution series as an AAP. More specifically, a dilution series of positive patient samples in the respective sample buffer from negative patient samples allows assessing the sensitivity of the optimized MRM assay in terms of $\mathrm{Ct}$ value. We made four such dilution series in four different matrices, now also including Virocult (a balanced salt solution containing antibiotics to inhibit the growth of any bacterial contaminants in the specimen) and Bioer (RNA preservative medium that enables viral inactivation). We selected four patients with a comparable in-house $\mathrm{Ct}$ value, and based on Figure $4 \mathrm{C}$, we assume that every $1 / 2$ dilution increases the $\mathrm{Ct}$ value by one. Figure 4D shows the LogSumAUC of all peptides because no ML has been done on Bioer and Virocult at this point. Most notably, the eSwab signal retains its linear correlation to $\mathrm{Ct}$ value down to a much lower signal, which is indicative of a lower background signal, i.e., lower noise and interference compared to the other media (Supplementary Data 19). In fact, when only the rank three peptides for Copan UTM$\mathrm{RT}$ and eSwab are used to present the results, the linearity in Copan UTM-RT persists, as well, albeit at a much shallower slope, implying that the ML possibly has selected for peptides with fewer interferences (Figure 4D inset).

Note that our in-house RT-PCR consistently has a higher $\mathrm{Ct}$ value in eSwab compared to the Copan UTM-RT (Figure 4C), and that the patient that was used for the eSwab turned out to have a large discrepancy between the in-house and clinical $\mathrm{Ct}$, even after a second run (red arrow in Figure 4E). Additionally, we have been presenting the strong correlation between $\mathrm{Ct}$ and LogSumAUC as a measure to assess the performance of an MRM, yet such correlation does not need to be linear in patient batches. In fact, a large discrepancy in the MRM signal between two patients with identical $\mathrm{Ct}$ values could have relevant biological grounds; for example, it might be an indication of an infectious or "superspreader" phenotype or correlate with the symptomatology and/or with (anticipated) severity of disease. We therefore argue against setting a specific $\mathrm{Ct}$ value as a threshold for MRM assay sensitivity and emphasize that the focus of interpretation of these results should be on the low noise level in eSwabs rather than the actual Ct values. Others have also avoided reporting such a direct link. ${ }^{21}$

In conclusion, a solid validation of this AAP is required before it can be universally adopted. In fact, measuring increasingly large patient cohorts, ideally along with documentation of (absence or presence of) symptoms could prove more useful to validate the performance of an MRM assay in a clinical setting.

Large Patient Cohort for Assessing Potential Assay Performance. As a final validation of the current performance of our Cov-MS assay, we analyzed 325 patient samples with the optimized conditions from the Cov-MS collaboration on a Xevo TQ-XS instrument (Waters Corporation) (Supplementary Data 20). To be able to compare the four different media in this batch, we prepared only $50 \mu \mathrm{L}$ of each sample, followed by precipitation and resuspension in $25 \mu \mathrm{L}$ digest buffer and 10 $\mu \mathrm{L}$ injections, i.e., $20 \mu \mathrm{L}$ medium equivalents on column. In parallel, we diagnosed 89 positive patients in the batch with our in-house RT-PCR. Remarkably, at lower Ct values, we consistently measured a higher than expected $\mathrm{Ct}$ value. At higher Ct values (>30), many of the patients did not even yield any signal for the $\mathrm{N}$ gene in-house (Figure $4 \mathrm{E}$ inset). This can be explained in part by the fact that the clinical RT-PCR was done on more samples. More specifically, the starting (190 vs $140 \mu \mathrm{L})$, elution $(40 \mu \mathrm{L}$ vs $60 \mu \mathrm{L})$, and final input volume ( 8 vs $5 \mu \mathrm{L}$ ) together indicate that our in-house assay only uses $30 \%$ of the volume in the PCR reaction, i.e., $\sim 10 \mu \mathrm{L}$, compared to the clinical assay, corresponding to nearly $2 \mathrm{Ct}$ values. Additionally, these samples had been preserved in the freezer at $-20{ }^{\circ} \mathrm{C}$ for over 4 months, and potentially mRNA degradation was prominent. Finally, low positive patients can be expected to be more prone to drop-out in the higher $\mathrm{Ct}$ range, i.e., after a larger number of amplification cycles, wherein error too can accumulate. Therefore, we only used patients with clinical $\mathrm{Ct}$ values below 30 as positive and with negative clinical diagnosis as 


\begin{tabular}{|c|c|c|c|c|c|c|}
\hline \multirow[t]{16}{*}{$A$} & $\begin{array}{c}/ 10 \mu l \\
\text { Ct levels Plasmid }\end{array}$ & $\begin{array}{c}/ \mathbf{1 0} \mu \mathrm{l} \\
\text { Number of genomes }\end{array}$ & $\begin{array}{l}300 \text { NCAP copies } \\
\text { per virion }\end{array}$ & $\begin{array}{c}/ 10 \mu l \\
\text { amol NCAP }\end{array}$ & C & $\begin{array}{l}\text { /320 } \mathrm{\mu l} \text { MRM (5Ct) } \\
\text { /Higher Measured Ct }\end{array}$ \\
\hline & 40 & 2.5 & $7.50 \mathrm{E}+02$ & 0.00 & & /Viral-like Particles \\
\hline & 39 & 5 & $1.50 E+03$ & 0.00 & & \\
\hline & 38 & 10 & $3.00 E+03$ & 0.00 & & \\
\hline & 37 & 20 & $6.00 \mathrm{E}+03$ & 0.01 & & \\
\hline & 36 & 40 & $1.20 E+04$ & 0.02 & & \\
\hline & 35 & 80 & $2.40 \mathrm{E}+04$ & 0.04 & & 40 \\
\hline & 34 & 160 & $4.80 \mathrm{E}+04$ & 0.08 & & 39 \\
\hline & 33 & 320 & $9.60 \mathrm{E}+04$ & 0.16 & & 38 \\
\hline & 32 & 640 & $1.92 \mathrm{E}+05$ & 0.32 & & 37 \\
\hline & 31 & 1280 & $3.84 \mathrm{E}+05$ & 0.64 & & 36 \\
\hline & 30 & 2560 & $7.68 \mathrm{E}+05$ & 1.28 & & 35 \\
\hline & 29 & 5120 & $1.54 \mathrm{E}+06$ & 2.55 & & 34 \\
\hline & 28 & 10240 & $3.07 E+06$ & 5.10 & & 33 \\
\hline & 27 & 20480 & $6.14 E+06$ & 10.20 & & 32 \\
\hline & 26 & 40960 & $1.23 E+07$ & 20.41 & & 31 \\
\hline \multirow[t]{7}{*}{ BEST CASE } & 25 & 81920 & $2.46 E+07$ & 40.81 & (iii) & 30 \\
\hline & 24 & 163840 & $4.92 \mathrm{E}+07$ & 81.62 & & 29 \\
\hline & 23 & 327680 & $9.83 E+07$ & 163.24 & (ii) & 28 \\
\hline & 22 & 655360 & $1.97 \mathrm{E}+08$ & 326.48 & & 27 \\
\hline & 21 & 1310720 & $3.93 E+08$ & 652.97 & & 26 \\
\hline & 20 & 2621440 & $7.86 \mathrm{E}+08$ & 1305.93 & & 25 \\
\hline & 19 & 5242880 & $1.57 \mathrm{E}+09$ & 2611.86 & (i) & 24 \\
\hline \multirow{10}{*}{ WORST CASE } & 18 & 10485760 & $3.15 E+09$ & 5223.73 & & 23 \\
\hline & 17 & 20971520 & $6.29 E+09$ & 10447.45 & & 22 \\
\hline & 16 & 41943040 & $1.26 \mathrm{E}+10$ & 20894.91 & & 21 \\
\hline & 15 & 83886080 & $2.52 E+10$ & 41789.81 & & 20 \\
\hline & 14 & 167772160 & $5.03 E+10$ & 83579.62 & & 19 \\
\hline & 13 & 335544320 & $1.01 \mathrm{E}+11$ & 167159.24 & & 18 \\
\hline & 12 & 671088640 & $2.01 \mathrm{E}+11$ & 334318.49 & & 17 \\
\hline & 11 & 1342177280 & $4.03 E+11$ & 668636.97 & & 16 \\
\hline & 10 & 2684354560 & $8.05 E+11$ & 1337273.94 & & 15 \\
\hline & 9 & 5368709120 & $1.61 \mathrm{E}+12$ & 2674547.88 & & 14 \\
\hline
\end{tabular}

B
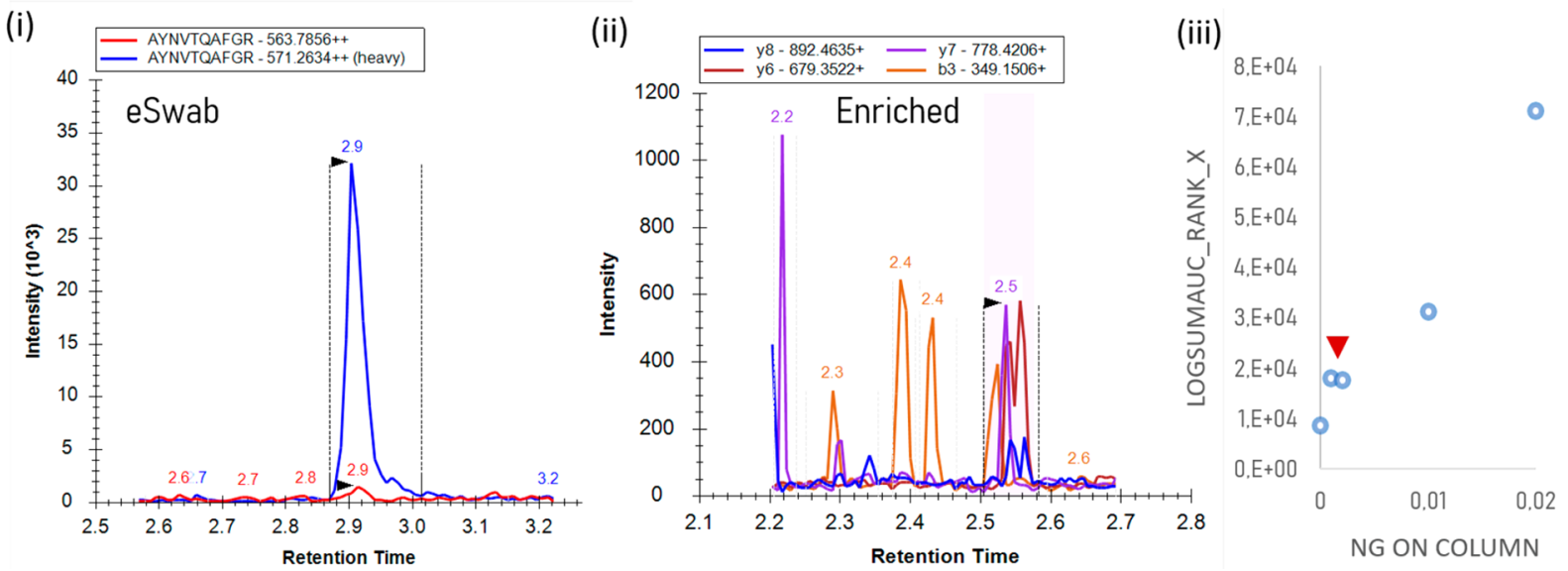

Figure 5. Projection of MRM capabilities. (A) Absolute quantities theoretically correlate $\mathrm{Ct}$ to the MRM assay. Ten genomes present in $10 \mu \mathrm{L}$ are detected by a Ct of 38 in perfect conditions (i.e., plasmids) with our in-house RT-PCR. Assuming 300 NCAP molecules per virion, a direct translation into attomoles is possible. The highlighted portion of the table depicts (B) three different limits of detection for the MRM assay. (i) AYNVTQAFGR with the Cov-MS QConCAT in eSwab background, (ii) following peptide enrichment (signal taken from in solution dilution from Figure 2B), and (iii) possibly by more data-driven approaches or summing all signal at the elution window of peptides without peak integration. (C) Potential practical correlation between $\mathrm{Ct}$ and MRM assay. By loading up to 32 times more by enrichment of targets could yield another $5 \mathrm{Ct}$ values of sensitivity. This is enforced by the fact that clinical RT-PCRs are expected to be higher than on plasmids and the MRM signal could be higher because of VLPs. 
negative. Note that this high degree of variability between RTPCR assays again impairs an easy definition for specificity and sensitivity of the MRM assay based on a Ct threshold.

Figure 4E shows the final outcome of the MRM assay using the rank 3 peptides from Figure $3 \mathrm{G}$ on the remaining 82 positive patients, i.e., 37 positive patients in Copan UTM-RT, 30 patients in eSwab, 11 patients in Bioer, and 4 patients in Virocult. Fifty-six negative patients in $\mathrm{eSwab}^{22}$ and Copan UTM-RT ${ }^{33}$ were subjected to the same manual peak boundary curation to define the background signal. Notably, the Cov-MS QConCAT construct was not detectable in all samples because the standard turned out to be underloaded at $1.25 \mathrm{ng}$ on column and was only used to facilitate a manual setting of peak boundaries. Thus, 138 out of the 325 patients were used in the final data analysis of the large patient cohort. Raw data of all runs are available and browsable on the Skyline Panorama server (https://panoramaweb.org/CovMS.url). As an alternative to setting a hard $\mathrm{Ct}$ threshold, the minimum background threshold is highlighted for Copan UTM-RT and eSwab, and the intersection with the linear regression of the positive patients is projected onto the $\mathrm{Ct}$ axis. This is the $\mathrm{Ct}$ above which the background, i.e. matrix, becomes too high to distinguish signal from noise, and we currently consider this to be the theoretical maximum sensitivity using the current workflow. All patient samples were analyzed in a randomized sample list (Supplementary Data 20), and at least some of the background outliers of eSwab could be attributed to the fact that they were run closely following a Copan UTM-RT sample and were subject to carry-over. In conclusion, background, i.e., matrix and not instrumental limitations, is currently the limiting factor for increasing the sensitivity.

We conclude that nonproteinaceous buffers are the only matrices that allow easy adaptation of the assay and to diagnose patients with $\mathrm{Ct}$ values above 20 without matrix removal. In fact, we strongly argue against the use of the protein-laden Copan UTM-RT for MS-based SARS-CoV-2 diagnosis given the reasons mentioned before. In fact, adopting a transport medium without protein or salts, which coordinately inactivates the virus, like $70 \%$ ethanol, would greatly facilitate sample processing and potentially improve sensitivity. In brief, the maximum attainable single-shot MRM would thus start with direct ice-cold acetone precipitation ( 7 volumes) on $175 \mu \mathrm{L}$ of saline medium in $1.5 \mathrm{~mL}$ Eppendorf vials, resuspending this in $35 \mu \mathrm{L}$ of $50 \mathrm{mM}$ TEABC, $0.5 \mu \mathrm{g}$ of trypsin/LysC, and 5\% acetonitrile with $10 \mathrm{ng}$ of CovMS QConCAT standard, digesting for $15 \mathrm{~min}$ at $37^{\circ} \mathrm{C}$, followed by addition of $3.5 \mu \mathrm{L}$ of $10 \%$ formic acid (end concentration $1 \%$ ) and-possibly following SPE-injecting $10 \mu \mathrm{L}$ on column. This is $45.5 \mu \mathrm{L}$ medium equivalent on column, or 2.25 times more than the patient batch analyzed here. While it is currently not proven that increasing the loading increases sensitivity-as the matrix background increases accordingly - we are confident that Cov-MS has strong potential of becoming a valuable addition to the diagnostic SARS-CoV-2 toolbox in the near future.

Cov-MS Digital Incubator: Facilitating Improvements for Clinical Roll-Out. Taken together, the high degree of variation in matrices and instrumentation was intercepted in this study by collaboration in the Cov-MS consortium. Uniquely, both academic and industrial partners have shown inspiring openness in a joint effort to quickly extend testing capacity and help alleviate pressure on current testing facilities. As this is possibly the first peptide-centric viral detection assay to be rolled-out in the clinic on a large scale, a lot of different perspectives on the best workflow exist in parallel, and collaboration allows one to quickly assess the best performing steps. Currently, such a collaborative effort is also driving the clinical roll-out in the Moonshot project in the $\mathrm{UK}^{43}$ which in turn is based on the Cov-MS assay and initiatives like COVIDMSC. ${ }^{25}$ Also in The Netherlands, institutionally driven efforts have been initiated. In order to facilitate further exchange of information, we therefore propose to persist the collaborative nature of this effort and we invite all interested parties with MS capabilities to join the Cov-MS Digital Incubator Microsoft Teams environment, so they can access all information gathered during this initial phase of development and can parallelize further efforts to improve applicability, robustness, and sensitivity of the assay. Current template threats are available for Waters Corporation, Sciex, Thermo, Shimadzu, Bruker, Agilent, and PerkinElmer. Access can be requested by sending name and affiliation to CovMS@ugent.be.

\section{FUTURE PERSPECTIVES}

We provided a detailed description of a community effort to build a robust and sensitive orthogonal assay to diagnose SARSCoV-2 positivity. To be able to track and align with the millions of RT-PCR tests that are performed daily, a collective effort by the scientific community is paramount, not in the least because MRM assays have rarely been used before to detect viruses. ${ }^{18}$ Therefore, we invite other laboratories to build on this platform and to introduce further optimizations across all parts of the workflow to help translate this assay into mainstream clinical laboratories and even to potentially start adding other pathogens to the assay. Here, we list some of the most pertinent issues and propose some potential solutions.

\section{Increasing Sensitivity}

Figure 5A displays the calculations that allow to discuss future improvements in terms of absolute numbers of genomes. Briefly, a perfect RT-PCR assay (as was done in-house on plasmids, Supplementary Data 18) detects 10 genomes in $10 \mu \mathrm{L}$ at a Ct of 38 , i.e., 2.5 genomes at $\mathrm{Ct} 40$. With every virion carrying $300-$ 350 NCAP molecules, ${ }^{33}$ the absolute amount of NCAP (in attomole) in $10 \mu \mathrm{L}$ is easily calculated. Therefore, the question becomes what can still be detected by current MS instruments. We distinguish three different thresholds (Figure 5B): (i) the signal attained in the current assay, i.e., in eSwab background with a QconCAT for efficient peak picking of low individual signals, (ii) pure peptides, i.e., how individual signals could look like following purification, and (iii) the last evidence we found of potential signal by LogSumAUC without individual peak calling on a Xevo TQ-S (Figure 2C,D and Supplementary Data 21). The latter shows what even more sensitive instruments like the Xevo TQ-XS (Waters Corporation) or the 7500 System (Sciex) could potentially still see as a peak. Still, Figure 5C depicts increased $\mathrm{Ct}$ thresholds to illustrate what could happen if target peptides are first enriched, and up to $320 \mu \mathrm{L}$ is used in a single shot (32 times more, i.e., $5 \mathrm{Ct}$ values). Other factors can also contribute to a shift in $\mathrm{Ct}$ values compared to the absolute number of genomes: (i) patient $\mathrm{Ct}$ values can be higher in clinical laboratories compared to the perfect plasmid situation and/or (ii) the potential presence of viral-like particles could boost the signal of an MRM assay. Based on these calculations, we predict that a clinical Ct value of 30 will most probably be the maximum attainable for current mass spectrometry instruments.

The Cov-MS laboratories have shown that the latest generation tandem quadrupole instruments are sensitive enough to detect patients passed a Ct 20 in the medium. In eSwab, a 
LOD of NCAP of at least $0.1 \mathrm{ng}$ on column (40 amol) was attained when the Cov-MS QConCAT was used for peak picking on the latest generation instruments, i.e., a 7500 from Sciex (Supplementary Data 21), in line with our earlier LODs depicted in Figure 2C. However, moving beyond $\mathrm{Ct}$ values of 25 - as implied before on high-resolution platforms ${ }^{10,39,44}$ — will thus require enrichment. Protein or peptide immuno-enrichment can be done either through antibodies (e.g., SISCAPA) or aptamers. ${ }^{45,46}$ This way, the few molecules that are present in a sample can be measured almost perfectly, i.e., in isolation with minimal interference. In the process, this will open the way to other COVID-19 sampling matrices, like gargle solution, saliva, and plasma, and finally alleviate the pressure from ever-changing media being used in the clinic. Together with the Cov-MS SIL standard that ascertains the correct detection of low signals and a low coefficient of variation, this could lead to an automatable, robust, and sensitive alternative for RT-PCR. Finally, enrichment strategies are insensitive to dilution. More specifically, it was recently suggested that for Belgium a population-wide weekly screening could be possible if patients are pooled by $32 .{ }^{47}$ This is the equivalent of lowering sensitivity by $5 \mathrm{Ct}$ values for RT-PCR but with SISCAPA enrichment; no loss in signal would be expected.

Will this be enough to become clinically relevant? Well, the limit of contagiousness was previously estimated to be close to Ct 33 . $^{48}$

\section{Increasing Throughput}

A major drawback of the current LC-MS based workflow is that data acquisition cannot be parallelized on a single instrument, contrary to RT-PCR assays that use multiwell plates. Yet, using 8 min gradients, one instrument can already measure over 150 patients/day and by multiplexing LC pumps this can be increased to $\sim 500$ per day ${ }^{21}$. One member of the consortium (Alderley Analytical) additionally looked at the application of 2 min gradients and showed that these should be feasible when the matrix is relatively clean (Supplementary Data 15), increasing the throughput to 600 patients per instrument per day without parallelization. With prior immuno-enrichment like SISCAPA, gradients as low as $1 \mathrm{~min}$ are within reach, as our preliminary results show (Supplementary Data 22 and Figure S2). At least from the sample preparation perspective, there is no reason why robotics cannot be deployed, as is the case for RT-PCR preparation.

One disruptive recent technology to further increase throughput is acoustic MS. In the implementation from Sciex (EchoMS), nanoliter droplets are acoustically ejected from the sample and into the instrument at a rate of $3 \mathrm{~Hz}$, with each droplet measuring up to five transitions. ${ }^{49}$ In time, this could lead to sampling an estimated 30000 patients a day per instrument. Likewise, MALDI-ToF instruments could provide very high throughput following immuno-enrichment of target peptides.

\section{Screening Multiple Pathogens in a Single Assay}

Using the proposed workflow, it is relatively straightforward to add mutations or even other pathogens for screening in a single assay. These include other corona viruses as well as influenza. This will greatly increase the impact of protein detection by MRM in the clinic.

\section{Making Data Analysis Clinically Applicable}

To cope with all the resulting data, the automation of data analysis in a clinically applicable way will still require some efforts. For the development of the assay, the use of Skyline enabled the parallel processing of all the consortium data, but in a clinical setting, vendor-specific software is more likely to be implemented. However, with recent advances in machine learning in the proteomics field, we are optimistic that a community effort in data analysis can move forward very quickly. ${ }^{50}$ This includes automated diagnosis based on the MRM data. Initiatives such as EuBIC, prominent advocates of data sharing, provide the perfect basis for seeding such efforts. Meeting diagnostic assay criteria required by regulatory agencies, including calibrators/internal standards, sample preparation, instrumentation, as well as data review and reporting, all mentioned and detailed here, requires consideration from a broader deployment perspective. Fortunately, however, these requirements are understood, as previous efforts such as the National Cancer Institute's Clinical Proteomic Technologies for Cancer initiative and the HUPO Proteomics Standards Initiative have aided the acceptance and validation processes by introducing the "verification" concept using MRM technologies, similarly to the Accreditation Standards concept and results introduced in this article. Together, this illustrates that collaboration will be key to the final success of a "fit-forpurpose" MRM assay. ${ }^{51}$

In conclusion, we describe the full pipeline for developing an MS-based assay for viral presence directly on clinical samples and with conventional instrumentation. The current assay allows the diagnosis of up to 200 COVID-19 patients per instrument per day with high viral load. However, our results are the stepping stone to a second generation assay wherein by removing matrix with peptide immunopurification, 1 min per patient would result in up to nearly 1500 patients of potentially up to $\mathrm{Ct} 30$ on a single LC-MS setup. Sample preparation for protein detection can be done under $\$ 5$ worth of reagents. Therefore, if 1000 patients per instrument can be reached, the cost of the instrument would soon be covered. Importantly, however, most clinical laboratories already use tandem quadrupole instruments in neonatal diagnostics. As opposed to PCR, MS can thus be deployed for many other clinical applications once the pandemic cools down. For sake of pandemic readiness, we need orthogonal diagnostics to PCR and LCMS is proving a very promising addition to that toolbox. To make it into the clinic, however, free sharing of all acquired data will be essential to intercept matrix and instrumental heterogeneity. At the same time, we cannot know for sure when this protein-based MSbased test will be ready for the clinic or when the clinic will be ready for a protein-based mass spectrometry test. Nevertheless, it is clear that mass spectrometry stands a chance of matching PCR's output and economies of scale, if a collective effort by the community is applied. The UK is currently setting the scene, building on collaborative efforts like COVID-MSC ${ }^{25}$ and CovMS and others will most likely follow. What is certain is that not trying would be scientifically negligent given the critical importance of the issue.

\section{MATERIALS AND METHODS}

\section{Sample Preparation}

In the discovery phase (blue), $50 \mu \mathrm{L}$ of a UTM-RT patient sample was precipitated by adding $450 \mu \mathrm{L}$ ( 9 volumes) of ice-cold acetone $(-20$ ${ }^{\circ} \mathrm{C}$ ). After spinning at $16000 \mathrm{~g}$ and $0{ }^{\circ} \mathrm{C}$, the supernatant was discarded and $1 \mu \mathrm{g}$ of trypsin/Lys-C mix (Promega) in $60 \mu \mathrm{L} 500 \mathrm{mM}$ triethylammonium bicarbonate (TEABC) buffer was added. This was followed by an incubation step of $4 \mathrm{~h}$ at $37^{\circ} \mathrm{C}$ to facilitate trypsin digestion. Next, $20 \mu \mathrm{L}$ of this sample was prepared for analysis in a final 
concentration of $0.1 \%$ formic acid of which $2 \mu \mathrm{L}$ was injected into the LC-MS system. In the consortium phase (red), the protocol was validated on a dilution series of two recombinant COVID-19 proteins NCAP_SARS2 and SPIKE_SARS2 (Sino Biological, Beijing, China) which were found to be most abundant in public data on SARS-CoV$2 .^{17,23}$ A triplicate dilution series of $250 \mu \mathrm{L}$ of negative patient Copan UTM-RT medium was spiked with different amounts of recombinant protein $(500,100,50,10,5,1,0.5,0.1,0.05,0 \mathrm{ng})$ resulting in a concentration range of $1 \mathrm{ng}$ to $300 \mathrm{fg}$ on column. Within the Cov-MS consortium, multiple sample preparation protocols were tested (see Detailed Methods section), which led to the following protocol for the clinical phase (green). A direct ice-cold acetone precipitation (7 volumes) on $175 \mu \mathrm{L}$ of medium in $1.5 \mathrm{~mL}$ Eppendorf Protein LoBind tubes, resuspending this in $35 \mu \mathrm{L} 50 \mathrm{mM}$ TEABC $0.5 \mu \mathrm{g}$ trypsin/LysC and $5 \%$ acetonitrile with $10 \mathrm{ng}$ of Cov-MS QConCAT standard, digesting for $15 \mathrm{~min}$ at $37^{\circ} \mathrm{C}$, followed by addition of $3.5 \mu \mathrm{L}$ of $10 \%$ formic acid (end concentration 1\%) and-possibly following SPEinjecting $10 \mu \mathrm{L}$ on column. The preliminary peptide immunopurification (SISCAPA) data (Supplementary Data 22) were obtained by performing the protocol described by Razavi et al. ${ }^{46}$ using polyclonal antibodies targeting two NCAP peptides: AYNVTQAFGR and DGIIWVATEGALNTPK.

\section{Data Acquisition}

For each peptide, the cone and collision energies were optimized within 8 min runs as described in the SOP (Supplementary Data 12). A scheduled MRM file was created, retaining the most sensitive peptides on each instrumental platform based on human inspection. Instrumental parameters are given in more detail in the Supporting Information, Detailed Methods section. Vendor-specific application notes are available online. ${ }^{35-38}$

\section{Data Analysis}

The data were analyzed in Skyline Daily (version 20.1.9.234) using a template file containing the 17 target peptides. Peak boundaries were manually adjusted, as this was required, considering the amount of interfering transitions, originating from the matrix. Note that adjusting the peak boundaries could be considered as a subjective task which might introduce bias in the data analysis.

\section{Data Availability}

The mass spectrometry data generated during the current study are being shared through the Skyline Panorama Public interface for easy accessibility (https://panoramaweb.org/CovMS.url) and are being deposited to the ProteomeXchange Consortium via the PRIDE partner repository with data set identifier: PXD022550. The Supporting Information will be made available through the Microsoft Teams group: "Cov-MS Digital Incubator”. Please send an email to covms@ugent.be to obtain access.

\section{Code Availability}

$\mathrm{MS}^{2} \mathrm{PIP}$, DeepLC, EncyclopeDIA, and the Machine Learning algorithms are open source, licensed under the Apache-2.0 License, and are hosted on https://github.com/compomics/ms2pip_c, https:// github.com/compomics/DeepLC, https://bitbucket.org/searleb/ encyclopedia/wiki/Home, and https://github.com/compomics/CovMS-scoring.

\section{ASSOCIATED CONTENT}

\section{Supporting Information}

The Supporting Information is available free of charge at https://pubs.acs.org/doi/10.1021/jacsau.1c00048.

Additional figures to highlight the variants of concern for the NCAP_SARS2 protein and some preliminary SISCAPA data using a $1 \mathrm{~min}$ LC gradient are included; the detailed methods section describes the different steps (sample preparation, data acquisition, data analysis, etc.) in the Cov-MS consortium in more detail (PDF)
Supplementary data (no skyline files/raw data) is available in the ZIP folder (ZIP)

\section{AUTHOR INFORMATION}

\section{Corresponding Author}

Maarten Dhaenens - ProGenTomics, Laboratory of Pharmaceutical Biotechnology, Ghent University, 9000 Ghent, Belgium; 이이.org/0000-0002-9801-3509;

Email: maarten.dhaenens@ugent.be

\section{Authors}

Bart Van Puyvelde - ProGenTomics, Laboratory of Pharmaceutical Biotechnology, Ghent University, 9000 Ghent, Belgium

Katleen Van Uytfanghe - Laboratory of Toxicology, Department of Bioanalysis, Faculty of Pharmaceutical Sciences, Ghent University, 9000 Ghent, Belgium

Olivier Tytgat - ProGenTomics, Laboratory of Pharmaceutical Biotechnology, Ghent University, 9000 Ghent, Belgium; Department of Life Science Technologies, Imec, 3000 Leuven, Belgium

Laurence Van Oudenhove - Waters Corporation, 2600 Antwerp, Belgium

Ralf Gabriels - VIB-UGent Center for Medical Biotechnology, VIB, 9000 Ghent, Belgium; Department of Biomolecular Medicine, Ghent University, 9000 Ghent, Belgium; ( ) orcid.org/0000-0002-1679-1711

Robbin Bouwmeester - VIB-UGent Center for Medical Biotechnology, VIB, 9000 Ghent, Belgium; Department of Biomolecular Medicine, Ghent University, 9000 Ghent,

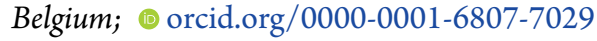

Simon Daled - ProGenTomics, Laboratory of Pharmaceutical Biotechnology, Ghent University, 9000 Ghent, Belgium

Tim Van Den Bossche - VIB-UGent Center for Medical Biotechnology, VIB, 9000 Ghent, Belgium; Department of Biomolecular Medicine, Ghent University, 9000 Ghent, Belgium; (1) orcid.org/0000-0002-5916-2587

Pathmanaban Ramasamy - VIB-UGent Center for Medical Biotechnology, VIB, 9000 Ghent, Belgium; Department of Biomolecular Medicine, Ghent University, 9000 Ghent, Belgium; Interuniversity Institute of Bioinformatics in Brussels, ULB/VUB, 1050 Brussels, Belgium; 이이.org/00000003-4039-5230

Sigrid Verhelst - ProGenTomics, Laboratory of Pharmaceutical Biotechnology, Ghent University, 9000 Ghent, Belgium

Laura De Clerck - ProGenTomics, Laboratory of Pharmaceutical Biotechnology, Ghent University, 9000 Ghent, Belgium

Laura Corveleyn - ProGenTomics, Laboratory of Pharmaceutical Biotechnology, Ghent University, 9000 Ghent, Belgium

Sander Willems - ProGenTomics, Laboratory of Pharmaceutical Biotechnology, Ghent University, 9000 Ghent, Belgium

Nathan Debunne - Drug Quality and Registration Group, Faculty of Pharmaceutical Sciences, Ghent University, 9000 Ghent, Belgium; (1) orcid.org/0000-0001-5839-7539

Evelien Wynendaele - Drug Quality and Registration Group, Faculty of Pharmaceutical Sciences, Ghent University, 9000 Ghent, Belgium 
Bart De Spiegeleer - Drug Quality and Registration Group, Faculty of Pharmaceutical Sciences, Ghent University, 9000 Ghent, Belgium; (1) orcid.org/0000-0002-6103-3565

Peter Judak - Doping Control Laboratory, Department of Diagnostic Sciences, Ghent University, 9000 Ghent, Belgium

Kris Roels - Doping Control Laboratory, Department of Diagnostic Sciences, Ghent University, 9000 Ghent, Belgium

Laurie De Wilde - Doping Control Laboratory, Department of Diagnostic Sciences, Ghent University, 9000 Ghent, Belgium; ○ orcid.org/0000-0002-3472-2170

Peter Van Eenoo - Doping Control Laboratory, Department of Diagnostic Sciences, Ghent University, 9000 Ghent, Belgium

Tim Reyns - Department of Clinical Chemistry, Ghent University Hospital, 9000 Ghent, Belgium

Marc Cherlet - Department of Pharmacology, Toxicology, and Biochemistry, Faculty of Veterinary Medicine, Ghent University, 9000 Ghent, Belgium

Emmie Dumont - Research Institute for Chromatography (RIC), 8500 Kortrijk, Belgium

Griet Debyser - Research Institute for Chromatography (RIC), 8500 Kortrijk, Belgium

Ruben t'Kindt - Research Institute for Chromatography (RIC), 8500 Kortrijk, Belgium

Koen Sandra - Research Institute for Chromatography (RIC), 8500 Kortrijk, Belgium

Surya Gupta - VIB-UGent Center for Medical Biotechnology, VIB, 9000 Ghent, Belgium; Department of Biomolecular Medicine, Ghent University, 9000 Ghent, Belgium; (1) orcid.org/0000-0002-6290-6161

Nicolas Drouin - Division of Systems Biomedicine and Pharmacology, Leiden Academic Centre for Drug Research, Leiden University, 2311 G Leiden, The Netherlands

Amy Harms - Division of Systems Biomedicine and Pharmacology, Leiden Academic Centre for Drug Research, Leiden University, 2311 G Leiden, The Netherlands

Thomas Hankemeier - Division of Systems Biomedicine and Pharmacology, Leiden Academic Centre for Drug Research, Leiden University, 2311 G Leiden, The Netherlands; ○ orcid.org/0000-0001-7871-2073

Donald J. L. Jones - Leicester Cancer Research Centre, RKCSB, University of Leicester, U.K., and John and Lucille van Geest Biomarker Facility, Cardiovascular Research Centre, Glenfield Hospital, Leicester LE1 7RH, United Kingdom; (1) orcid.org/ 0000-0001-6583-870X

Pankaj Gupta - The Department of Chemical Pathology and Metabolic Diseases, Level 4, Sandringham Building, Leicester Royal Infirmary, Leicester LE1 7RH, United Kingdom

Dan Lane - The Department of Chemical Pathology and Metabolic Diseases, Level 4, Sandringham Building, Leicester Royal Infirmary, Leicester LE1 7RH, United Kingdom

Catherine S. Lane - AB Sciex, Macclesfield SK10 4TG, United Kingdom

Said El Ouadi - AB Sciex, Macclesfield SK10 4TG, United Kingdom

Jean-Baptiste Vincendet - AB Sciex, Macclesfield SK10 4TG, United Kingdom

Nick Morrice - AB Sciex, Macclesfield SK10 4TG, United Kingdom

Stuart Oehrle - Waters Corporation, Milford, Massachusetts 01757, United States

Nikunj Tanna - Waters Corporation, Milford, Massachusetts 01757, United States; (1) orcid.org/0000-0001-7770-7651
Steve Silvester - Alderley Analytical, Macclesfield SK10 4TG, United Kingdom

Sally Hannam - Alderley Analytical, Macclesfield SK10 4TG, United Kingdom

Florian C. Sigloch - Polyquant GmbH, 93077 Bad Abbach, Germany; (1) orcid.org/0000-0001-8130-6885

Andrea Bhangu-Uhlmann - Polyquant GmbH, $93077 \mathrm{Bad}$ Abbach, Germany

Jan Claereboudt - Waters Corporation, 2600 Antwerp, Belgium

N. Leigh Anderson - SISCAPA Assay Technologies, Inc., Washington, D.C. 20009, United States

Morteza Razavi - SISCAPA Assay Technologies, Inc., Washington, D.C. 20009, United States

Sven Degroeve - VIB-UGent Center for Medical Biotechnology, VIB, 9000 Ghent, Belgium; Department of Biomolecular Medicine, Ghent University, 9000 Ghent, Belgium

Lize Cuypers - Clinical Department of Laboratory Medicine, UZ Leuven, 3000 Leuven, Belgium

Christophe Stove - Laboratory of Toxicology, Department of Bioanalysis, Faculty of Pharmaceutical Sciences, Ghent University, 9000 Ghent, Belgium; 이이.org/0000-00017126-348X

Katrien Lagrou - Clinical Department of Laboratory Medicine, UZ Leuven, 3000 Leuven, Belgium

Geert A. Martens - AZ Delta Medical Laboratories, AZ Delta General Hospital, 8800 Roeselare, Belgium

Dieter Deforce - ProGenTomics, Laboratory of Pharmaceutical Biotechnology, Ghent University, 9000 Ghent, Belgium

Lennart Martens - VIB-UGent Center for Medical Biotechnology, VIB, 9000 Ghent, Belgium; Department of Biomolecular Medicine, Ghent University, 9000 Ghent, Belgium; (1) orcid.org/0000-0003-4277-658X

Johannes P. C. Vissers - Waters Corporation, Milford, Massachusetts 01757, United States; (1) orcid.org/00000001-6283-8456

Complete contact information is available at: https://pubs.acs.org/10.1021/jacsau.1c00048

Notes

The authors declare the following competing financial interest(s): L. Van Oudenhove, J. Claereboudt, S. Oehrle, N. Tanna, and J. P. C. Vissers are employed by Waters Corporation. C. S. Lane, S. El Ouadi, J.-B. Vincendet, and N. Morrice are employed by Sciex. F. Sigloch and A. Bhangu-Uhlmann are employed by Polyquant GmbH. M. Razavi and L. Anderson are employed by SISCAPA.

\section{ACKNOWLEDGMENTS}

This research was funded by grants from the Research Foundation Flanders (FWO): Grant No. 11B4518N to B.V.P., Grant No. $1 \mathrm{~S} 50918 \mathrm{~N}$ to R.G., Grant No. $1 \mathrm{~S} 90918 \mathrm{~N}$ to T.V.D.B., Grant No. 3S031319 to S.V., Grant No. G032816N to P.R., Grant No. G042518N to L.M., 1 S21017N to N.D., and $12 \mathrm{E} 9716 \mathrm{~N}$ to M.D. Ph.D. grants from the University Ghent Special Research Funding (BOF) awarded to O.T. (Grant No. BOF18/DOC/200) and L.C. (Grant No. BOF20/DOC/220); a BOF-COVID-19 project grant (01C01920) from Ghent University; and by the European Union's Horizon 2020 Programme under Grant Agreement 823839 (H2020-INFRAIA-2018-1). Ethical approval was obtained from the Ethics 
Committees of University Hospital Leuven (S63879) and University Hospital Ghent (BC-09263).

\section{REFERENCES}

(1) OECD. Testing for COVID-19: A way to lift confinement restrictions 2020, May, 1-21; https://www.oecd.org/coronavirus/policyresponses/testing-for-covid-19-a-way-to-lift-confinement-restrictions89756248/ (accessed March 29, 2021).

(2) Surkova, E.; Nikolayevskyy, V.; Drobniewski, F. False-positive COVID-19 results: hidden problems and costs. Lancet Respir. Med. 2020, 8 (12), 1167.

(3) FDA. SARS-CoV-2 Reference Panel Comparative Data; https:// www.fda.gov/medical-devices/coronavirus-covid-19-and-medicaldevices/sars-cov-2-reference-panel-comparative-data.

(4) ASM. ASM Expresses Concern about Coronavirus Test Reagent Shortages, 2020; https://asm.org/Articles/Policy/2020/March/ASMExpresses-Concern-about-Test-Reagent-Shortages\#:-:text= ASM\%20Expresses\%20Concern\%20About\%20Coronavirus\%20Tes t\%20Reagent $\% 20$ Shortages,-March\%2010\%2C\%202020\&text= Clinical\%20microbiologists\%20and\%20clinical\%20labs,the\%20respon s e $\% 20$ t o \% 20 C O V I D \% 2 D 19 . \& t e x t =

We\%20applaud\%20the\%20CDC\%20for,specimen\%20swab\%20instea d\%20of\%20two (accessed March 29, 2021).

(5) CEBM. Are you infectious if you have a positive PCR test result for COVID-19? 2020; https://www.cebm.net/covid-19/infectiouspositive-pcr-test-result-covid-19/ (accessed March 29, 2021).

(6) Atkinson, B; Petersen, E. SARS-CoV-2 shedding and infectivity. Lancet 2020, 395, 1339-40.

(7) Nachtigall, F. M.; Pereira, A.; Trofymchuk, O. S.; Santos, L. S. Detection of SARS-CoV-2 in nasal swabs using MALDI-MS. Nat. Biotechnol. 2020, 38, 1168.

(8) Mak, G. C.; Cheng, P. K.; Lau, S. S.; Wong, K. K.; Lau, C.; Lam, E. T.; Chan, R. C.; Tsang, D. N. Evaluation of rapid antigen test for detection of SARS-CoV-2 virus. J. Clin. Virol. 2020, 129, 104500.

(9) Bezstarosti, K.; Lamers, M. M.; Haagmans, B. L.; Demmers, J. A. A.. Targeted Proteomics for the Detection of SARS-CoV-2 Proteins. bioRxiv 2020, DOI: 10.1101/2020.04.23.057810

(10) Renuse, S.; Vanderboom, P. M.; Maus, A. D.; Kemp, J. V.; Gurtner, K. M.; Madugundu, A. K.; et al. Development of mass spectrometry-based targeted assay for direct detection of novel SARSCoV-2 coronavirus from clinical specimens. medRxiv 2020, DOI: $10.1101 / 2020.08 .05 .20168948$.

(11) Ihling, C.; Tänzler, D.; Hagemann, S.; Kehlen, A.; Hüttelmaier, S.; Sinz, A. Mass Spectrometric Identification of SARS-CoV-2 Proteins from Gargle Solution Samples of COVID-19 Patients. J. Proteome Res. 2020, 19 (11), 4389.

(12) Giri, R.; Bhardwaj, T.; Shegane, M.; Gehi, B. R.; Kumar, P.; Gadhave, K.; et al. When Darkness Becomes a Ray of Light in the Dark Times: Understanding the COVID-19 via the Comparative Analysis of the Dark Proteomes of SARS-CoV-2, Human SARS and Bat SARS-Like Coronaviruses. bioRxiv 2020, DOI: 10.1101/2020.03.13.990598.

(13) Grenga, L.; Gallais, F.; Pible, O.; Gaillard, J.-C.; Gouveia, D.; Batina, H.; Bazaline, N.; Ruat, S.; Culotta, K.; Miotello, G.; Debroas, S.; Roncato, M.-A.; Steinmetz, G.; Foissard, C.; Desplan, A.; Alpha-Bazin, B.; Almunia, C.; Gas, F.; Bellanger, L.; Armengaud, J. Shotgun proteomics of SARS-CoV-2 infected cells and its application to the optimization of whole viral particle antigen production for vaccines. Emerging Microbes Infect. 2020, 9, 1712.

(14) Ortea, I.; Bock, J.-O. Re-analysis of SARS-CoV-2 infected host cell proteomics time-course data by impact pathway analysis and network analysis. A potential link with inflammatory response. Aging 2020, 12, 11277.

(15) Gordon, D. E.; Jang, G. M.; Bouhaddou, M.; Xu, J.; Obernier, K.; White, K. M.; O'Meara, M. J.; Rezelj, V. V.; Guo, J. Z.; Swaney, D. L.; Tummino, T. A.; Huttenhain, R.; Kaake, R. M.; Richards, A. L.; Tutuncuoglu, B.; Foussard, H.; Batra, J.; Haas, K.; Modak, M.; Kim, M.; Haas, P.; Polacco, B. J.; Braberg, H.; Fabius, J. M.; Eckhardt, M.; Soucheray, M.; Bennett, M. J.; Cakir, M.; McGregor, M. J.; Li, Q.; Meyer, B.; Roesch, F.; Vallet, T.; Mac Kain, A.; Miorin, L.; Moreno, E.;
Naing, Z. Z. C.; Zhou, Y.; Peng, S.; Shi, Y.; Zhang, Z.; Shen, W.; Kirby, I. T.; Melnyk, J. E.; Chorba, J. S.; Lou, K.; Dai, S. A.; Barrio-Hernandez, I.; Memon, D.; Hernandez-Armenta, C.; Lyu, J.; Mathy, C. J. P.; Perica, T.; Pilla, K. B.; Ganesan, S. J.; Saltzberg, D. J.; Rakesh, R.; Liu, X.; Rosenthal, S. B.; Calviello, L.; Venkataramanan, S.; Liboy-Lugo, J.; Lin, Y.; Huang, X.-P.; Liu, Y.; Wankowicz, S. A.; Bohn, M.; Safari, M.; Ugur, F. S.; Koh, C.; Savar, N. S.; Tran, Q. D.; Shengjuler, D.; Fletcher, S. J.; O’Neal, M. C.; Cai, Y.; Chang, J. C. J.; Broadhurst, D. J.; Klippsten, S.; Sharp, P. P.; Wenzell, N. A.; Kuzuoglu-Ozturk, D.; Wang, H.-Y.; Trenker, R.; Young, J. M.; Cavero, D. A.; Hiatt, J.; Roth, T. L.; Rathore, U.; Subramanian, A.; Noack, J.; Hubert, M.; Stroud, R. M.; Frankel, A. D.; Rosenberg, O. S.; Verba, K. A.; Agard, D. A.; Ott, M.; Emerman, M.; Jura, N.; von Zastrow, M.; Verdin, E.; Ashworth, A.; Schwartz, O.; d'Enfert, C.; Mukherjee, S.; Jacobson, M.; Malik, H. S.; Fujimori, D. G.; Ideker, T.; Craik, C. S.; Floor, S. N.; Fraser, J. S.; Gross, J. D.; Sali, A.; Roth, B. L.; Ruggero, D.; Taunton, J.; Kortemme, T.; Beltrao, P.; Vignuzzi, M.; Garcia-Sastre, A.; Shokat, K. M.; Shoichet, B. K.; Krogan, N. J. A SARS-CoV-2-Human Protein-Protein Interaction Map Reveals Drug Targets and Potential Drug-Repurposing. Nature 2020, 583, 459-468.

(16) Orsburn, B. C.; Jenkins, C.; Miller, S. M.; Neely, B. A.; Bumpus, N. N. In silico approach toward the identification of unique peptides from viral protein infection: Application to COVID-19. SSRN J. 2020, DOI: $10.2139 /$ ssrn.3589835.

(17) Davidson, A. D.; Williamson, M. K.; Lewis, S.; Shoemark, D.; Carroll, M. W.; Heesom, K. J.; Zambon, M.; Ellis, J.; Lewis, P. A.; Hiscox, J. A.; Matthews, D. A. Characterisation of the transcriptome and proteome of SARS-CoV-2 using direct RNA sequencing and tandem mass spectrometry reveals evidence for a cell passage induced in-frame deletion in the spike glycoprotein that removes the furin-like cleavage site. Genome Med. 2020, 12, 68.

(18) Grossegesse, M.; Hartkopf, F.; Nitsche, A.; Schaade, L.; Doellinger, J.; Muth, T. Perspective on Proteomics for Virus Detection in Clinical Samples. J. Proteome Res. 2020, 19, 4380.

(19) Neuman, B. W.; Buchmeier, M. J. Supramolecular Architecture of the Coronavirus Particle. Advances in Virus Research; Academic Press Inc., 2016; pp 1-27.

(20) Swann, H.; Sharma, A.; Preece, B.; Peterson, A.; Eldridge, C.; Belnap, D. M.; Vershinin, M.; Saffarian, S. Minimal system for assembly of SARS-CoV-2 virus like particles. Sci. Rep. 2020, 10, 21877.

(21) Cardozo, K. H. M.; Lebkuchen, A.; Okai, G. G.; Schuch, R. A.; Viana, L. G.; Olive, A. N.; Lazari, C. d. S.; Fraga, A. M.; Granato, C. F. H.; Pintao, M. C. T.; Carvalho, V. M. Establishing a mass spectrometrybased system for rapid detection of SARS-CoV-2 in large clinical sample cohorts. Nat. Commun. 2020, 11 (1), 6201.

(22) Plante, J. A.; Liu, Y.; Liu, J.; Xia, H.; Johnson, B. A.; Lokugamage, K. G.; Zhang, X.; Muruato, A. E.; Zou, J.; Fontes-Garfias, C. R.; Mirchandani, D.; Scharton, D.; Bilello, J. P.; Ku, Z.; An, Z.; Kalveram, B.; Freiberg, A. N.; Menachery, V. D.; Xie, X.; Plante, K. S.; Weaver, S. C.; Shi, P.-Y. Spike mutation D614G alters SARS-CoV-2 fitness. Nature 2021, 592 (7852), 116.

(23) Bojkova, D.; Klann, K.; Koch, B.; Widera, M.; Krause, D.; Ciesek, S.; Cinatl, J.; Munch, C. SARS-CoV-2 infected host cell proteomics reveal potential therapy targets. Nature 2020, 583, 469.

(24) Pino, L. K.; Searle, B. C.; Bollinger, J. G.; Nunn, B.; MacLean, B.; MacCoss, M. J. The Skyline ecosystem: Informatics for quantitative mass spectrometry proteomics. Mass Spectrometry Reviews; John Wiley and Sons Inc., 2020; Vol. 39, pp 229-244.

(25) Struwe, W.; Emmott, E.; Bailey, M.; Sharon, M.; Sinz, A.; Corrales, F. J. The COVID-19 MS Coalition-accelerating diagnostics, prognostics, and treatment. The Lancet; Lancet Publishing Group, 2020; Vol. 395, pp 1761-1762.

(26) Perez-Riverol, Y.; Csordas, A.; Bai, J.; Bernal-Llinares, M.; Hewapathirana, S.; Kundu, D. J; Inuganti, A.; Griss, J.; Mayer, G.; Eisenacher, M.; Perez, E.; Uszkoreit, J.; Pfeuffer, J.; Sachsenberg, T.; Yılmaz, S.; Tiwary, S.; Cox, J.; Audain, E.; Walzer, M.; Jarnuczak, A. F; Ternent, T.; Brazma, A.; Vizcaino, J. A. The PRIDE database and related tools and resources in 2019: Improving support for quantification data. Nucleic Acids Res. 2019, 47, D442. 
(27) Van Puyvelde, B.; Willems, S.; Gabriels, R.; Daled, S.; De Clerck, L.; Vande Casteele, S.; Staes, A.; Impens, F.; Deforce, D.; Martens, L.; Degroeve, S.; Dhaenens, M. Removing the Hidden Data Dependency of DIA with Predicted Spectral Libraries. Proteomics 2020, 20 (3-4), No. 1900306.

(28) WHO. Laboratory biosafety guidance related to coronavirus disease (COVID-19), 2020; https://www.who.int/publications/i/ item/laboratory-biosafety-guidance-related-to-coronavirus-disease(covid-19) (accessed March 29, 2021).

(29) Zecha, J.; Lee, C. Y.; Bayer, F. P.; Meng, C.; Grass, V.; Zerweck, J. Data, Reagents, Assays and Merits of Proteomics for SARS-CoV-2 Research and Testing. Mol. Cell Proteomics 2020, 19 (9), 1503-22.

(30) Gouveia, D.; Grenga, L.; Gaillard, J.; Gallais, F.; Bellanger, L.; Pible, O.; et al. Shortlisting SARS-CoV-2 Peptides for Targeted Studies from Experimental Data-Dependent Acquisition Tandem Mass Spectrometry Data. Proteomics 2020, 20 (14), 2000107.

(31) PyMOL, https://pymol.org/2/ (accessed March 29, 2021).

(32) Slechtová, T.; Gilar, M.; Kalíková, K.; Tesařová, E. Insight into Trypsin Miscleavage: Comparison of Kinetic Constants of Problematic Peptide Sequences. Anal. Chem. 2015, 87, 7636.

(33) Yao, H.; Song, Y.; Chen, Y.; Wu, N.; Xu, J.; Sun, C.; Zhang, J.; Weng, T.; Zhang, Z.; Wu, Z.; Cheng, L.; Shi, D.; Lu, X.; Lei, J.; Crispin, M.; Shi, Y.; Li, L.; Li, S. Molecular Architecture of the SARS-CoV-2 Virus. Cell 2020, 183, 730.

(34) Maarten Dhaenens. Cov-MS Consortium Launch (Teams Meeting). 2020.

(35) Van Oudenhove, L.; Tanna, N.; Claereboudt, J.; Vissers, H.; Van Puyvelde, B.; Daled, S. Comprehending COVID-19: Multiple Reaction Monitoring Transition Selection and Optimization Strategies for LCMS Based SARS-CoV-2 Detection; https://www.waters.com/ nextgen/mx/es/library/application-notes/2020/comprehendingcovid-19-multiple-reaction-monitoring-transition-selection-andoptimization-strategies-for-lc-ms-based-sars-cov-2-detection.html (accessed March 29, 2021).

(36) Van Oudenhove, L.; Claereboudt, J.; Moore, R.; Vissers, H.; Van Puyvelde, B.; Daled, S. Comprehending COVID-19: Maximizing LCMS Detection Dynamic Range for Multiple Reaction Monitoring Based SARS-CoV-2 Analysis; https://www.waters.com/waters/library. htm?locale $=$ en US\&lid=135075039 (accessed March 29, 2021).

(37) Oehrle, S.; Van Oudenhove, L.; Claereboudt, J.; Vissers, H.; Van Puyvelde, B.; Daled, S. Comprehending COVID-19: Application of UniSpray and Electrospray Ionization for the Detection of Proteolytic Digested SARS-CoV-2 Proteins; https://www.waters.com/waters/ library.htm?locale $=$ en_US\&lid $=135077658$ (accessed March 29, 2021).

(38) Lane, C. S.; Van Puyvelde, B.; Van Uytfanghe, K.; Dhaenens, M. Targeted assay for quantification of proteins from the SARS-CoV-2 coronavirus Using the SCIEX Triple Quad ${ }^{\mathrm{TM}} 5500+$ System-QTRAP Ready. 2020; https://sciex.com/content/dam/SCIEX/pdf/technotes/all/MRM-peptide-quant-assay-covid19-proteins.pdf (accessed March 29, 2021).

(39) Gouveia, D.; Miotello, G.; Gallais, F.; Gaillard, J.-C.; Debroas, S.; Bellanger, L.; Lavigne, J.-P.; Sotto, A.; Grenga, L.; Pible, O.; Armengaud, J. Proteotyping SARS-CoV-2 Virus from Nasopharyngeal Swabs: A Proof-of-Concept Focused on a 3 min Mass Spectrometry Window. J. Proteome Res. 2020, 19, 4407.

(40) Beynon, R. J.; Doherty, M. K.; Pratt, J. M.; Gaskell, S. J. Multiplexed absolute quantification in proteomics using artificial QCAT proteins of concatenated signature peptides. Nat. Methods 2005, 2 (8), 587-9.

(41) Holman, S. W.; Mclean, L.; Eyers, C. E. RePLiCal: A QconCAT Protein for Retention Time Standardization in Proteomics Studies. 2016; https://pubs.acs.org/sharingguidelines (accessed March 29, 2021).

(42) Peaks of light and heavy peptides have unequal peak boundaries. MacCoss Lab Software 2020; https://skyline.ms/announcements/ home/support/thread.view? rowId=48979 (accessed March 29, 2021).

(43) Gurdasani, D.; Bear, L.; Bogaert, D.; Burgess, R. A; Busse, R.; Cacciola, R.; Charpak, Y.; Colbourn, T.; Drury, J.; Friston, K.; Gallo, V.;
Goldman, L. R; Greenhalgh, T.; Hyde, Z.; Kuppalli, K.; Majumder, M. S; Martin-Moreno, J. M; McKee, M.; Michie, S.; Mossialos, E.; Nouri, A.; Pagel, C.; Pimenta, D.; Popescu, S.; Priesemann, V.; Rasmussen, A. L; Reicher, S.; Ricciardi, W.; Rice, K.; Silver, J.; Smith, T. C; Wenham, C.; West, R.; Yamey, G.; Yates, C.; Ziauddeen, H. The UK needs a sustainable strategy for COVID-19. Lancet 2020, 396, 1800.

(44) Nikolaev, E. N.; Indeykina, M. I.; Brzhozovskiy, A. G.; Bugrova, A. E.; Kononikhin, A. S.; Starodubtseva, N. L.; Petrotchenko, E. V.; Kovalev, G. I.; Borchers, C. H.; Sukhikh, G. T. Mass-Spectrometric Detection of SARS-CoV-2 Virus in Scrapings of the Epithelium of the Nasopharynx of Infected Patients via Nucleocapsid N Protein. J. Proteome Res. 2020, 19, 4393.

(45) Zhang, L.; Fang, X.; Liu, X.; Ou, H.; Zhang, H.; Wang, J.; Li, Q.; Cheng, H.; Zhang, W.; Luo, Z. Discovery of sandwich type COVID-19 nucleocapsid protein DNA aptamers. Chem. Commun. 2020, 56, 10235.

(46) Razavi, M.; Leigh Anderson, N.; Pope, M. E.; Yip, R.; Pearson, T. $W$. High precision quantification of human plasma proteins using the automated SISCAPA Immuno-MS workflow. New Biotechnol. 2016, 33, 494.

(47) Libin, P.; Willem, L.; Verstraeten, T.; Torneri, A.; Vanderlocht, J.; Hens, N. Assessing the feasibility and effectiveness of householdpooled universal testing to control COVID-19 epidemics. PLoS Comput. Biol. 2021, 17, e1008688.

(48) La Scola, B.; Le Bideau, M.; Andreani, J.; Hoang, V. T.; Grimaldier, C.; Colson, P.; Gautret, P.; Raoult, D. Viral RNA load as determined by cell culture as a management tool for discharge of SARSCoV-2 patients from infectious disease wards. Eur. J. Clin. Microbiol. Infect. Dis. 2020, 39 (6), 1059-61.

(49) Echo MS.SCIEX; https://sciex.com/products/integratedsolutions/Echo-ms.html

(50) Bouwmeester, R.; Gabriels, R.; Van Den Bossche, T.; Martens, L.; Degroeve, S. The Age of Data-Driven Proteomics: How Machine Learning Enables Novel Workflows. Proteomics 2020, 20, 1900351.

(51) Carr, S. A.; Abbatiello, S. E.; Ackermann, B. L.; Borchers, C.; Domon, B.; Deutsch, E. W.; et al. Targeted peptide measurements in biology and medicine: Best practices for mass spectrometry-based assay development using a fit-for-purpose approach. Mol. Cell Proteomics 2014, 13 (3), 907-17. 